\title{
On three-dimensional self-avoiding walk symmetry classes
}

\author{
A Rechnitzer and A L Owczarek \\ Department of Mathematics and Statistics, The University of Melbourne, Victoria 3010, Australia \\ E-mail: andrewr@ms.unimelb.edu.au and aleks@ms.unimelb.edu.au
}

Received 18 October 1999, in final form 2 February 2000

\begin{abstract}
In two dimensions the universality classes of self-avoiding walks (SAWs) on the square lattice, restricted by allowing only certain two-step configurations (TSCs) to occur within each walk, has been argued to be determined primarily by the symmetry of the set of allowed rules. In three dimensions early work tentatively found one (undirected) universality class different to that of unrestricted SAWs on the simple cubic lattice. This rule was a natural generalization of the square lattice 'spiral' SAW to three dimensions. In this report we examine a variety of three-dimensional SAW models with different step restrictions, carefully chosen so as to search for a connection between the symmetry of the rules and possible new universality classes. A first analysis of the scaling of the radius of gyration suggests several universality classes, including the one found earlier, and perhaps some novel class(es). However, a classification of these universality classes using the symmetries of the rules, or other basic rule properties, is not evident. Further analysis of the number of configurations and moment of inertia tensor suggests that in three dimensions the only non-trivial or undirected universality class is that of unrestricted SAWs.
\end{abstract}

\section{Introduction}

The self-avoiding walk (SAW) (see, for example, [1] and references therein) and its derivatives have been a major source of models describing the thermodynamic, geometric and topological properties of different types of long chain polymers in solution. A large number of modifications, such as the addition of various interactions (e.g. surface or intra-polymer) or particular restrictions (e.g. directedness), have been made to the basic model to mimic either various physical situations or to allow for easier analysis (such as exact solution). Some of these changes in the basic model modify the scaling behaviour of system properties, and hence change the universality class. For example, it is well known that restricting SAWs on the square or cubic lattices by only allowing steps in the positive axial directions, thus producing so-called directed (or rather fully directed) walks (see [2,3]), changes the way that the radius of gyration scales with polymer length. Geometric restrictions, such as directedness or spirality, are one particular type of modification whose effect on the scaling behaviour of walks is of considerable interest. Non-directed but restricted walks were first introduced by Grassberger [4] and the models he examined were found to be in the same universality class as SAWs. However, spiral SAWs (SSAWs) [5-8] on the square lattice were subsequently found to be in a different universality class to unrestricted SAWs (and, of course, directed walks (DWs)). Another novel universality class, studied subsequently [9-13], is that of anisotropic SSAWs (ASSAWs), also defined on the square lattice. This class has proven difficult to analyse [13] but appears to be distinct from the other classes. 
The SAW models previously analysed that give rise to the various universality classes of walks with geometric constraints can be described by the title 'two-step-restricted walks' (TSRWs) since these models are specified by the directions in which subsequent steps are allowed after steps in each of the four (square lattice) lattice directions are made. For example, one might specify that after either positive $x$ - or $y$-axis steps only positive $x$ - or $y$-axis steps can be made and that negative steps are disallowed - this gives the fully DW. Such rules might model oriented polymers in complicated external fields. We note here that these TSRW models are by their nature oriented. In two dimensions a wide-ranging study [12] of the universality classes, as determined by the scaling of the mean square end-to-end distance, of two-step-restricted SAWs on the square lattice, without interactions, has been made. Apart from cataloguing the universality classes in two dimensions, and analysing the ASSAW class further, this study elucidated the relationship between the symmetries of the lattice models and their universality classes. The study found that symmetry was a major factor in deciding into which universality class a rule's configurations would fall.

The theoretical understanding of the effect of geometric restrictions on the scaling behaviour of three-dimensional SAWs is less well understood. Guttmann and Wallace [14] introduced two walk models on the simple cubic lattice, which they called model S and model A. Model A was argued to be in the same universality class as three-dimensional SAWs, while the model $\mathrm{S}$, a three-dimensional equivalent of the spiral walk, appeared to be a member of a distinct class.

In this work we examine a subset of the possible two-step restriction rule models on the simple cubic lattice in a manner similar to that of Guttmann et al [12]. Our purpose in doing this is twofold: firstly to determine the possible universality classes for such rules in three dimensions and secondly to attempt to find a similar relationship between the symmetry of the microscopic rule and the macroscopic scaling behaviour of the ensemble of walk configurations, as in the work of Guttmann et al [12]. We focus on rules that are likely to produce configurations that are not simply directed, or zero, one- or two-dimensional. Exact enumeration and subsequent series analysis has been the basis of our studies here. Our first analyses on the radius of gyration series show the possibility of novel universality classes in three dimensions. However, no correspondence can be made between the symmetry of the rules and these apparent universality classes. Also, the difference in the exponents between different universality classes is relatively small. More detailed analysis paints a different picture: that there are no 'real' equivalents of the spiral or anisotropic spiral classes among three-dimensional two-step rules - that is, there are no novel universality classes-and so we deduce that the unique topology of two dimensions must be an important factor in determining the number of different universality classes there.

We begin our discussion in the next section with the definition of a TSRW model. The space of TSRW models is much larger in three dimensions than in two. To understand the factors important in our choice of models to study we first review the results of earlier square lattice studies with a view to extracting the salient features. We provide a complete pictorial classification of the interesting square lattice TSRW models in appendix A. This motivates the cubic lattice models we have studied, which we describe in section 2 also. We then describe the generation of our exact enumeration data and its various analyses in section 3. Finally, in section 4 we provide a discussion of our numerical results, summarizing the different reasonable theoretical scenarios, and cautiously pointing out the most likely conclusions. 


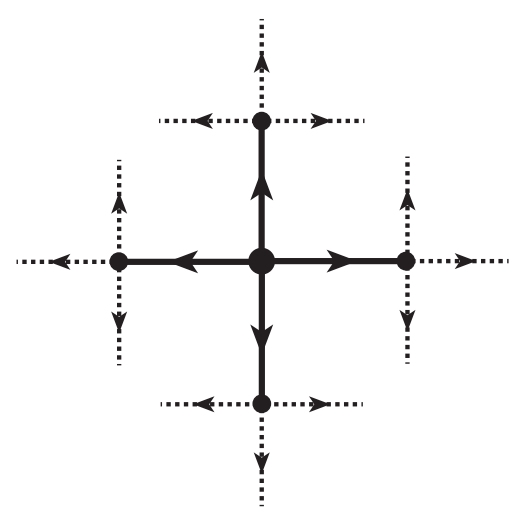

(a)

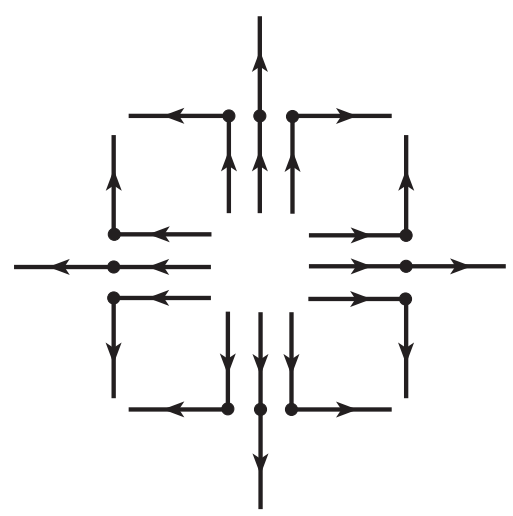

(b)

Figure 1. The construction of the allowed TSCs in a TSRW starts in $(a)$ with the consideration of each of the possible bonds (full lines) of a vertex. One specifies which of the next steps (dashed lines) is allowed. Shown here also in $(b)$ are the 12 square lattice TSCs.

\section{Two-step-restricted walk models}

\subsection{Definition}

In this paper we consider two-step-restricted SAW (TSRW) models on the square and simple cubic lattices. However, this type of model can be defined on any lattice with a finite number of types of vertices. To be specific, and for the sake of simplicity, let us first consider the square lattice problem. One begins by considering oriented SAWs on this lattice (starting from some fixed origin). To specify the model one does two things. The first is to generate a set of allowed two-step configurations. To do this one considers a vertex of the lattice and, in turn, each of the bonds emanating from that site. On the square lattice there are four bonds emanating from each site. Assuming a step of the walk is on the bond (one of the four) under consideration one then specifies the bonds that are allowed for the next step of the walk. From each of the four first-step bonds there are three possible continuing bonds (considering self-avoidance). This means there are 12 possible TSCs for an oriented SAW on the square lattice. Figure 1 illustrates this construction with the possible 12 TSCs explicitly given. To specify a TSRW model one must say which of the 12 two-step configurations are allowed and which are not allowed. There are hence $2^{12}=4096$ possible rules, and so 4096 walk models. Figure 2 illustrates one TSRW rule with the associated allowed TSCs also shown. The second part in obtaining a model is to take all oriented SAWs on the lattice where each two-step segment of each walk is one of the allowed TSCs. One then can ignore the orientation and this leaves a set of SAWs which then defines a two-step-restricted rule model.

Now, as mentioned above, this construction can be made on any regular lattice. On a $d$-dimensional hypercubic lattice the cardinality of the rule space is $2^{2 d(2 d-1)}$, so while in two dimensions the cardinality of the rule space is $2^{12}=4096$, in three dimensions it is $2^{30}=1073741824$. The size of the three-dimensional rule space leads us first to re-examine the two-dimensional rule space more carefully. 


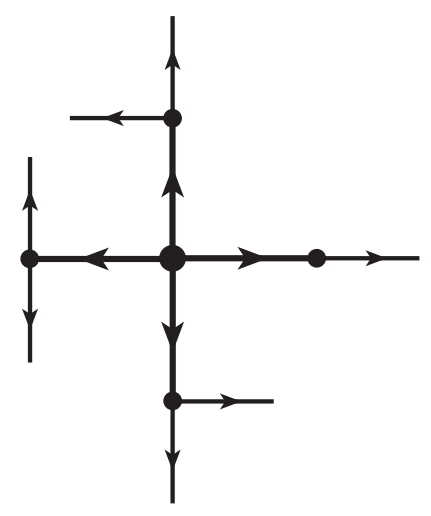

(a)

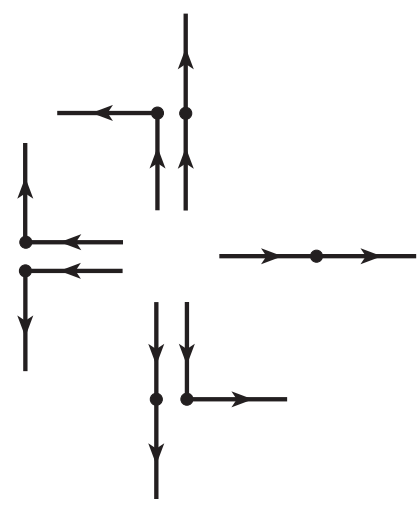

(b)

Figure 2. A particular TSRW rule is illustrated in $(a)$ with the associated allowed two-step configurations shown in $(b)$.

\subsection{Two dimensions}

2.2.1. Summary of two-dimensional results. On the square lattice Guttmann et al [12] have catalogued the classes of two-step-restricted SAWs, and examined the relationship between the scaling of the size of the objects, as measured by the various components of the end-to-end distance, and the symmetries of the walk rule. Some SAW models on other two-dimensional lattices have also been considered previously. Most notably the unrestricted SAW on the triangular and honeycomb lattices [15-17] appear, within error calculations, to be in the same universality class as square lattice SAWs. Variants of the spiral SAWs on the triangular lattice have also been studied [18-20]. At least with respect to the scaling of the size of the walks, as measured by the radius of gyration for example, the triangular and square lattice SAWs show similar scaling behaviour.

In Guttmann et al [12] the universality class was delineated mainly by considering the mean square end-to-end distance $\left\langle R_{e}^{2}\right\rangle_{n}$ scaling of these walk models with walk length $n$, this being a measure of the size of the model polymer similar in behaviour to the radius of gyration, $\left\langle R_{g}^{2}\right\rangle_{n}$. The exponent associated with any measure of the average area of configurations, $\left\langle R^{2}\right\rangle_{n}$, is denoted $v$ and one usually expects the dominant asymptotic form to be

$$
\left\langle R^{2}\right\rangle_{n} \sim A n^{2 v} \quad \text { as } n \rightarrow \infty .
$$

For SAWs without restriction in two dimensions it is expected that $v=\frac{3}{4}$ [21], regardless of lattice. To be most general one needs to define scaling exponents in the maximal and minimal scaling directions, that is $v_{\|}$and $v_{\perp}$ respectively. The work of Guttmann et al [12] concluded that there are seven universality classes of two-step restriction models on the square lattice delineated by their 'size' scaling. Three of these are such that neither of the exponents take on the values 1 or 0 (these values imply some kind of one- or zero-dimensional behaviour respectively). These three classes are: unrestricted SAWs with $v_{\|}=v_{\perp}=\frac{3}{4}$; SSAWs where $v_{\|}=v_{\perp}=\frac{1}{2}[8,18]$ with confluent multiplicative logarithmic factors in the asymptotic form; and ASSAWs where the latest Monte Carlo evidence [13] suggests $v_{\|}=2 v_{\perp}=0.95(2)$. To be precise, the scaling form $[8,18]$ for SSAW geometric size has been derived exactly as

$$
\left\langle R^{2}\right\rangle_{n} \sim A_{S p} n(\log n)^{2} \quad \text { as } n \rightarrow \infty .
$$

The ASSAW model has certainly been the hardest to characterize and this may also be because of the existence of confluent logarithms in the scaling form for $\left\langle R^{2}\right\rangle_{n}$ for that class [13]. There 
Table 1. The scaling of the number of configurations, $c_{n}$, for several two-dimensional walk models.

\begin{tabular}{lll}
\hline $\begin{array}{l}\text { Walk } \\
\text { model }\end{array}$ & $\begin{array}{l}\text { Scaling form } \\
\text { for the number of walks }\end{array}$ & $\begin{array}{l}\text { Exponent and constant } \\
\text { values or estimates }\end{array}$ \\
\hline SAW & $c_{n} \sim B \mu^{n} n^{\gamma-1}$ & $\gamma=\frac{43}{32}[21]$ \\
Square spiral & $c_{n} \sim B \mathrm{e}^{\frac{2 \pi}{\sqrt{3}} \sqrt{n}} n^{\beta}$ & $\beta=-\frac{7}{4}[5-8]$ \\
Triangular spiral I & $c_{n} \sim B \mathrm{e}^{\pi \sqrt{\frac{2}{3}} \sqrt{n}} n^{\beta}$ & $\beta=-\frac{5}{4}[18]$ \\
Triangular spiral II and III & $c_{n} \sim B \mathrm{e}^{2 \pi \sqrt{n}} \log \left(\frac{n}{12}\right) n^{\beta}$ & $\beta=-\frac{13}{4}[20]$ \\
ASSAW & $c_{n} \sim B \mu^{n} \mathrm{e}^{a \sqrt{n}} n^{\beta}$ & $\beta \approx 0.9, a \approx 0.14[11]$ \\
DW & $c_{n} \sim B \mu^{n}$ & $\mu>1$ and $\gamma=1[22]$ \\
\hline
\end{tabular}

is no apparent exact solution for any ASSAW rule as there is for the SSAW class. A point worth noting here is that the radius of gyration seems to be affected by smaller corrections-to-scaling than the mean square end-to-end distance [13], and so exponent estimates obtained from the radius of gyration converge more quickly to a stable asymptotic value.

The classification of rules not falling into one of the three classes mentioned above is given by the following: some rules do not produce any long walks so $v_{\|}=0$ and $\nu_{\perp}=0$, that is, they are trivial or zero-dimensional rules; there are one-dimensional rules with $v_{\|}=1$ and $v_{\perp}=0$ where configurations are essentially made up of a single one-dimensional walk; there are rules that produce configurations made up of different one-dimensional walks, perhaps concatenated together a bounded number of times - they have $v_{\|}=1$ and $v_{\perp}=1$; finally, some rules give walks that fall into the universality class of DWs with $v_{\|}=1$ and $v_{\perp}=\frac{1}{2}$ (these are often described as $(1+1)$-dimensional or even ' $\frac{3}{2}$ '-dimensional).

The other property that has been commonly used to classify the behaviour of a SAW model is the scaling, or asymptotic form, of the number of configurations (or partition function), $c_{n}$, of length $n$. For SAWs it is usually expected that the dominant asymptotic form of $c_{n}$ is given by

$$
c_{n} \sim B \mu^{n} n^{\gamma-1} \quad \text { as } n \rightarrow \infty
$$

where $\mu$ is the 'connective' constant and $\gamma$ is the universal entropic critical exponent. However, the scaling forms of $c_{n}$ for SSAWs and ASSAWs have been shown, exactly in the case of SSAWs [5-8, 18], and predicted numerically in the case of ASSAWs [11], to have different forms. The two-dimensional results for $c_{n}$ are summarized in the table 1. Only in the case of unrestricted SAWs and DWs is it possible to interpret the power of the algebraic factor as a critical exponent (only in these cases does the associated generating function have a dominant algebraic singularity). The models labelled triangular spiral II and III have a multiplicative logarithmic confluent factor in their scaling form relative to the square lattice spirals. The geometric size scaling for these models remains undetermined so it is unclear how to interpret the triangular lattice results. It is likely that the geometric size scaling is less sensitive to minor variations in the model and that the exponent $v$ for these models is $\frac{1}{2}(\log )$ as in the case of square lattice spiral and 'triangular spiral I' walks.

2.2.2. Discussion of square lattice rules. We now concentrate on the classification of the square lattice TSRW models via their geometric scaling form. As described above, Guttmann et al [12] found that there are seven universality classes. They also attempted to ascertain the microscopic constraints on the rules that determine the geometric scaling form of the walks. They concluded that three factors were important in determining the universality class. The first 
Table 2. Length scale exponents and symmetries for the seven known two-dimensional universality classes of two-step-restricted rule SAW. We define a one letter code for each class. The letter ' $y$ ' stands for 'yes', 'n' for 'no', and 'e' for 'either'.

\begin{tabular}{llllll}
\hline Rule & $v_{\|}$ & $v_{\perp}$ & Rotation by $90^{\circ}$ & Rotation by $180^{\circ}$ & Reflection \\
\hline SAW $(S)$ & $\frac{3}{4}$ & $\frac{3}{4}$ & $\mathrm{e}$ & $\mathrm{y}$ & $\mathrm{y}$ \\
Spiral $(P)$ & $\frac{1}{2}(\log )$ & $\frac{1}{2}(\log )$ & $\mathrm{y}$ & $\mathrm{y}$ & $\mathrm{n}$ \\
Anisotropic spiral $(A)$ & $0.95(2)$ & $0.47(1)$ & $\mathrm{n}$ & $\mathrm{y}$ & $\mathrm{n}$ \\
Directed $(D)$ & 1 & $\frac{1}{2}$ & $\mathrm{n}$ & $\mathrm{e}$ & $\mathrm{e}$ \\
Pseudo-1D $(U)$ & 1 & 1 & $\mathrm{e}$ & $\mathrm{e}$ & $\mathrm{e}$ \\
1D $(O)$ & 1 & 0 & $\mathrm{n}$ & $\mathrm{e}$ & $\mathrm{e}$ \\
Trivial $(T)$ & 0 & 0 & $\mathrm{e}$ & $\mathrm{e}$ & $\mathrm{e}$ \\
\hline
\end{tabular}

was the (somewhat imprecise) idea that there must be enough of the 12 TSCs allowed to give a non-trivial or non-one-dimensional rule. Secondly, they quoted 'balance' as a criterion: rules that do not have equal numbers of continuing steps in the positive and negative components of each axis are either directed, one-dimensional or trivial. This criterion was tested for several rules and seems to be well borne out by the exact enumeration studies. One can also argue that if the rule is unbalanced then the random walk generated with the rule will be directed. Furthermore, one can plausibly conjecture that adding self-avoidance should not affect this directedness. Hence, unbalancedness is a good indication that the SAW generated with this rule is directed also. Note, however, that the converse is not true: the 'balance' condition is satisfied by some rules that give directed and one-dimensional walks. The final determining factor in the classification of the non-directed and non-one-dimensional (and trivial) rules was argued to be that of symmetry. The symmetries involved are single rotations and reflections: on the square lattice rotations by $\pi$ and $\frac{\pi}{2}$, and reflections about the lines $\theta=0, \frac{\pi}{4}, \frac{\pi}{2}, \frac{3 \pi}{4}$. In table 2 the seven (geometric scaling) universality classes are listed, along with the symmetries obeyed by the various rules in that class. So if one excludes directed, one- or zero-dimensional TSRW rules then:

- All SAW-like rules have rotation-by- $\pi$ symmetry and some reflection symmetry.

- All spiral-like rules have rotation-by- $\frac{\pi}{2}$ (and hence rotation-by- $\pi$ ) symmetry but no reflection symmetry.

- All ASSAW-like rules have rotation-by- $\pi$ symmetry but no rotation-by- $\frac{\pi}{2}$ or reflection symmetries.

To be able to tackle the three-dimensional TSRW models let us first consider this classification in a little more depth. The balance condition can be expanded. Since one can always obtain the same walk configurations of a particular rule by considering the 'reverse' rule, which is obtained by reversing the orientation on the set of TSCs (and reversing the origin of the walks), it is sensible to also enforce a balance condition on the reversed walk of any rule one requires to be non-directed or non-one-dimensional. We denote this the 'reverse-balance' condition. Note that there are rules that are balanced but not reverse-balanced and so produce such walks in the directed or one-dimensional universality classes (see, for example, rule $(\mathrm{k})$ in [12]). As mentioned previously, there are 4096 TSRW models on the square lattice. There are, however, only 80 such rules that obey the balance and reverse-balance conditions, which we shall refer to as the symmetric-balance condition from now on. For completeness we provide a complete catalogue of the 80 two-dimensional symmetric-balanced rules in appendix A, with classification according to the total number of continuing steps and universality class. The symmetries mentioned above mean that there are in fact only 24 distinct symmetric-balanced 
rules on the square lattice. ÔDistinct rulesÕ here implies that the set of walk configurations are distinct up to lattice symmetries.

However, there is clearly one further criterion missing to distinguish the 'interesting' rules. One can see in hindsight that none of the rules that are symmetric-balanced and fall into the $D$, $U, O$ or $T$ classes obey the following condition: that from each of the four directions one can by a sequence of allowed steps end up in any direction (including itself again) while obeying self-avoidance. We call this the mixing condition: it is, of course, related to the fact that there are 'enough' continuing steps in each direction.

So, following on from Guttmann et al [12] one can write down a simple set of rules to determine if a rule produces walks in one of the $S, P$ or $A$ classes. Starting with all TSRW rules:

(1) All rules that are not symmetric-balanced are directed, one-dimensional or trivial (that is in the $D, U, O$ or $T$ classes).

(2) All symmetric-balanced rules that are not 'mixing' rules are also in the $D, U, O$ or $T$ classes.

(3) All symmetric-balanced and mixing rules that have reflection symmetry about any axis are of the SAW $(S)$ universality class (expect possibly one rule which we call the anti-spiral model-see appendix A).

(4) All symmetric-balanced and mixing rules that do not have a reflection symmetry but are symmetric with respect to a rotation by $\frac{\pi}{2}$ are in the SSAW $(P)$ universality class.

(5) All symmetric-balanced and mixing rules that are neither reflection symmetric nor symmetric under a $\frac{\pi}{2}$ rotation are in the ASSAW $(A)$ class.

Note that all rules in the $S, P$ and $A$ classes (in fact, of the original 4096 there are only 22 rules - seven distinct rules - in these classes) with the exception of the rule mentioned in the appendix, called the anti-spiral, are symmetric with respect to rotations of $\pi$. (If the anti-spiral was in a novel universality class then the above classification would simply be expanded to distinguish the symmetric-balanced and mixing rules that have a reflection symmetry but no rotation symmetry at all.)

It is also interesting to note that one can distinguish the non-one-dimensional and non-DW by examination of their turning numbers. We define the turning number for a two-dimensional walk rule as the square of the difference of the number of TSCs that make a left turn to the number of TSCs that make a right turn. Those TSCs that proceed straight ahead make no contribution to the turning number. Using the turning number we could replace the last three steps in the above classification scheme by:

(3) $(S)$ rule walks have turning number 0 ,

(4) $(P)$ rule walks have turning number 16 and

(5) (A) rule walks have turning number 4.

The anti-spiral has turning number 0 .

So, in summary, the set of TSRW rules giving walks in the 'interesting' classes of $S, P$ and $A$ are distinguished from other rules by the symmetric-balance and mixing conditions, while they are distinguished from each other by the consideration of the symmetry of the rules. We note that it may be possible to distinguish rules in the $D, U, O$ or $T$ classes from each other but this is of less interest.

\subsection{Three dimensions: delineating properties of the two-step rule space}

In two dimensions the cardinality of the TSRW rule space is $2^{12}=4096$, while in three dimensions it is $2^{30}=1073741824$. In this paper we shall follow the lessons learned in 
the discussion of two-dimensional models described above. We do this by only considering rules that are symmetric-balanced and mixing. Let us call the set of TSRW rules that obey the symmetric-balanced and mixing conditions the symmetric-mixing rules. So let us consider the implications of these conditions for the space of three-dimensional TSRW rules.

2.3.1. Characterizing symmetric-balanced rules. On the simple cubic lattice there are 30 two-step configurations, so we could encode a particular rule by a 30 bit binary number. Alternatively we can encode the rules using a $6 \times 6$ square matrix of zeros and ones in the following way (six because vertices are sixfold coordinated on the simple cubic lattice). We label the lattice axes in the usual way with $x, y$ and $z$, and steps in the $\pm x$ as $r$ and $l$ respectively, steps in the $\pm y$ as $\boldsymbol{f}$ and $\boldsymbol{b}$ respectively, and steps in the $\pm z$ as $\boldsymbol{u}$ and $\boldsymbol{d}$ respectively. Hence a TSC made up of an 'up' step in the positive $z$ direction followed by a 'backward' step in the negative $y$ direction is labelled as $\boldsymbol{u b}$. We define the matrix, $\boldsymbol{M}$, as

$$
M=\left[\begin{array}{cccccc}
r r & 0 & r f & r b & r u & r d \\
0 & l l & l f & l b & l u & l d \\
f r & f l & f f & 0 & f u & f d \\
b r & b l & 0 & b b & b u & b d \\
u r & u l & u f & u b & u u & 0 \\
d r & d l & d f & d b & 0 & d d
\end{array}\right]
$$

where the elements are 1 or 0 depending on whether the corresponding TSCs occur in the rule space or not, respectively. That is, if the TSC $\boldsymbol{u b}$ occurs in our rule then the position $(5,4)$, labelled by $\boldsymbol{u} \boldsymbol{b}$ in equation (2.4) above, will contain a 1, otherwise it contains 0 . For general dimensional hypercubic lattices the matrix $M$ has binary elements with fixed zero elements for positions $(2 k-1,2 k)$ and $(2 k, 2 k-1)$ for all $k \in\{1, \ldots, d\}$.

We can write the various balance restrictions for the hypercubic lattice simply as follows: the balance condition requires that the sum of elements of $\boldsymbol{M}$ in successive columns taken in pairs is the same, that is

$$
\sum_{i} \boldsymbol{M}_{i,(2 k-1)}=\sum_{i} \boldsymbol{M}_{i, 2 k} \quad \text { for } \quad k \in\{1, \ldots, d\}
$$

while the reverse-balance condition requires that the sum of elements of $M$ in successive rows taken in pairs is the same, that is

$$
\sum_{j} \boldsymbol{M}_{(2 k-1), j}=\sum_{j} \boldsymbol{M}_{2 k, j} \quad \text { for } \quad k \in\{1, \ldots, d\} .
$$

The number of such rules can be calculated, and further, the detailed numbers of such rules made from a fixed number of TSCs can be calculated (see appendix B) by constructing a generating function that sums over all allowed matrices $M$ subject to the constraints (2.5) and (2.6) above. The above construction can be applied in any dimension and in appendix B we have calculated the total number of symmetric-balanced rules as

- 80 walk models in two dimensions,

- 432096 walk models in three dimensions and

- 478340593664 walk models in four dimensions.

This subset of symmetric-balanced two-step rules is manageable in two dimensions (we have in fact catalogued them completely in this paper). However, in three dimensions it is far too large to be examined in its entirety. 
2.3.2. Symmetries of TSRW rules in three dimensions. By restricting the consideration to only distinct symmetric-balanced rules the number of rules on the square lattice falls from 80 to 24 . If we further add mixing to the constraints imposed then this number falls to seven. So we now consider the symmetries of cubic lattice TSRW. This serves a dual purpose. The symmetries allow us to focus our attention on the distinct rules (rules that give distinct ensembles) and also provides a possible set of conditions that may be used to delineate any new universality classes discovered, as in two dimensions. We also consider the turning number as a property that may also delineate such classes.

We now list all single rotations and reflection symmetries of the rules, as well as defining what we call the turning number of the rule. We also define a symmetry we call flip-symmetry: this was considered since its existence is a quick way to ensure that the rule is symmetricbalanced. The properties we have used in three dimensions to attempt to classify TSRW rules are

(1) Rotational symmetries about the coordinate axes

- $\pm \frac{\pi}{2}$

- $\pi$.

(2) Reflection symmetries

- reflection in coordinate planes. The normals of these planes are given by: $\vec{n}=\hat{e}_{x}$, $\hat{e}_{y}, \hat{e}_{z} ;$ the unit vectors in each axial direction.

- reflection in diagonal planes†. The normals of these planes are given by: $\vec{n}=$ $\left(\hat{e}_{x} \pm \hat{e}_{y}\right),\left(\hat{e}_{x} \pm \hat{e}_{z}\right),\left(\hat{e}_{y} \pm \hat{e}_{z}\right)$.

(3) Turning number

- The turning number for a three-dimensional walk rule is the sum of the turning numbers of the planar walk rules in each co-ordinate plane when viewed from the positive side of the co-ordinate axis normal to that plane.

(4) Flip symmetry

- send each co-ordinate to its negative. In two dimensions this is equivalent to rotation by $\pi$. If a walk has the flip symmetry, then it is balanced and reverse-balanced. The reverse is not true.

It should be noted that it can be easily shown that TSRW rules in three dimensions with a $\frac{\pi}{2}$ rotational symmetry have a plane of reflection; the normal of the plane of reflection is the axis of the rotation. The converse is not true.

So in later discussion we attempt to classify the rules studied into universality classes according to which single symmetry operations acting on the rules leave the rules unchanged. All the rules (bar one) examined had the flip symmetry, and as noted above this implies all the rules were symmetric-balanced.

\subsection{Construction of symmetric-mixing cubic lattice TSRW rules}

Without actually going through all 432096 , or even the smaller number of distinct isosymmetric sets, of the symmetric-balanced TSRW rules on the cubic lattice we wanted to be

$\dagger$ The operator to reflect a vector in the plane with normal vector $\vec{n}=\left(\hat{e}_{x}+\hat{e}_{y}+\hat{e}_{z}\right)$, is given by the matrix:

$$
R_{\left(\hat{e}_{x}+\hat{e}_{y}+\hat{e}_{z}\right)}=\frac{1}{3}\left[\begin{array}{ccc}
1 & -2 & -2 \\
-2 & 1 & -2 \\
-2 & -2 & 1
\end{array}\right] .
$$

From this matrix we see that the vector $\hat{e}_{x}$ is mapped to $\frac{1}{3}\left(\hat{e}_{x}-2 \hat{e}_{y}-2 \hat{e}_{z}\right)$. That is, this operator is not closed on the vector space $\mathbb{Z}^{3}$. Hence a walk rule cannot have symmetry under this reflection, nor reflections in planes with normals given by $\left(\hat{e}_{x} \pm \hat{e}_{y} \pm \hat{e}_{z}\right)$. 
able to choose a set of three-dimensional rules that adequately explored this set of models (by encompassing the different symmetries, etc). We also wanted to ensure the mixing condition held. To do this we chose to consider walk rules in three dimensions such that on each of the three axial planes the rule was one of a small number of planar walks. We chose to construct rules using representatives from most of the two-dimensional universality classes. We chose from the following rules (see the catalogue of rules in appendix A for the rule numbers):

$S$ Rule 1-Unrestricted planar SAW: in class $(S)$, turning number 0;

3 Rule 5-Three-choice walks: in class $(A)$, turning number 4;

2 Rule 6(a)-Two-choice walks: in class 4(A), turning number 4;

$P$ Rule 1-Planar spiral walks: in class $(P)$, turning number 16;

$D$ Rule 7-DW (with reverse): in class $(D)$, turning number 0 ;

$C$ Rule 22-Walks that are concatenations of $1 \mathrm{~d}$ walks: in class $(U)$, turning number 0 ;

$O$ Rule 19-Walks that are one-dimensional along either axis: in class $(U)$, turning number 0;

$R$ Rule 17-Walks that form incomplete one-dimensional rectangles: in class $(O)$ turning number 4.

Each three-dimensional rule we considered can be given a three letter code such as $(P-O-3)$, which means the plane with a normal along the $z$-axis was given the square lattice rule $(P)$ while the plane with a normal along the $y$-axis was given the square lattice rule $(O)$ and the plane with a normal along the $x$-axis was given the square lattice rule (3). The three-dimensional SAW is denoted by $(S-S-S)$, Guttmann and Wallace's spiral walk [14] (model $S)$ is $(P-P-P)$ while Guttmann and Wallace's 'anisotropic spiral' 3D-equivalent (model A) is $(S-C-3)$. Note that not every combination of these types is possible; for example, one cannot construct a $(S-3-2)$ walk. This is because each quadratic lattice walk is defined by 12 choices of TSCs that is made, and since there are three axial planes we have a total of 36 choices to make. However, in three dimensions one really has only 30 possible choices to make, so some combinations will be inconsistent (and so impossible). The five models that we have analysed most extensively are illustrated in figures 3-7. In figure 3 the rule $(P-P-P)$ is shown, in figure 4 the rule $(P-2-2)$ is shown, in figure 5 the rule $(P-O-3)$ is shown, in figure 6 the rule $(S-C-P)$ is shown, while in figure 7 the rule $(P-R-2)$ is shown. Note that the turning number of the three-dimensional rule is simply the sum of the turning numbers of the three two-dimensional rules that make up the rule.

In fact we have examined by exact enumeration studies all the walk rules constructed plane-by-plane with the added restriction of not having more than one plane with a onedimensional or directed rule $(D, C, O$ or $R)$. This restriction was made to ensure we ended up with three-dimensional rules that were mixing. We considered the 38 such rules initially. All these rules had flip-symmetry and so were symmetric-balanced, and therefore symmetricmixing. This was still too many rules to consider in depth and because of the larger connective constants in three dimensions than in two the series enumerated were relatively shorter. We chose the nine most promising rules from a numerical point of view that covered many of the symmetry combinations plus three other rules (one not constructed in the above manner) so as to include all possible symmetry combinations. These rules included both the rules considered by Guttmann and Wallace previously [14].

We especially constructed a rule outside the gamut of the procedure described above so as to produce a rule that was symmetric under rotations by $\pi$ but not under rotations by $\frac{\pi}{2}$ or any reflection. We call this rule Rot- $\pi$ and it is a symmetric-balanced rule. The $M$ matrix for 


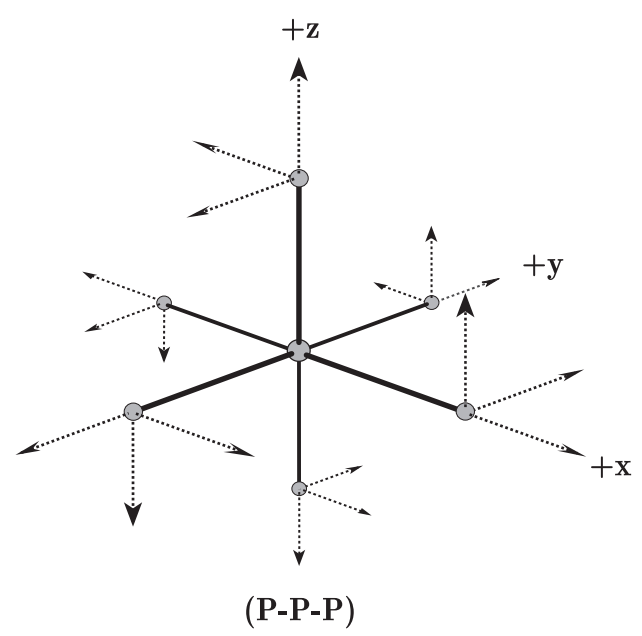

Figure 3. An illustration of the $(P-P-P)$ rule.

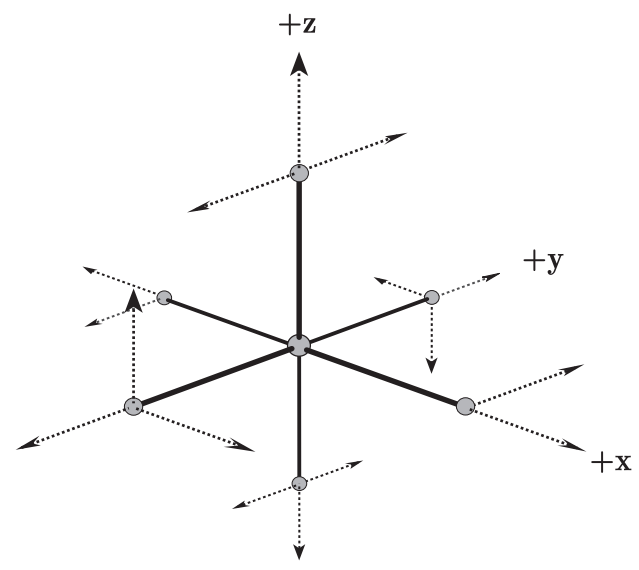

(P-3-T3)

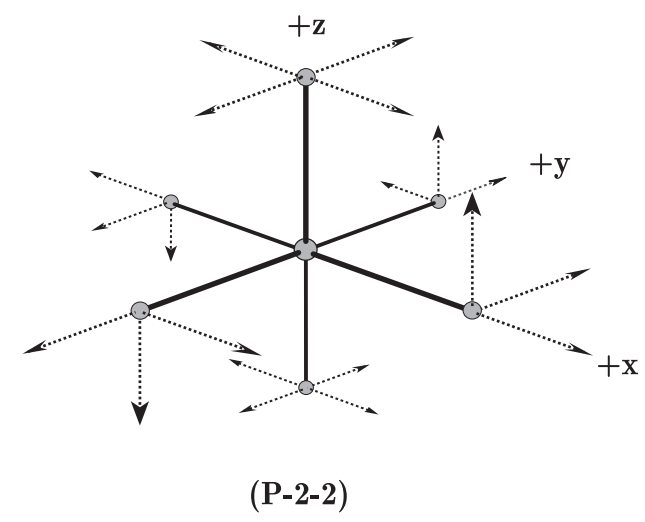

Figure 4. An illustration of the $(P-2-2)$ rule

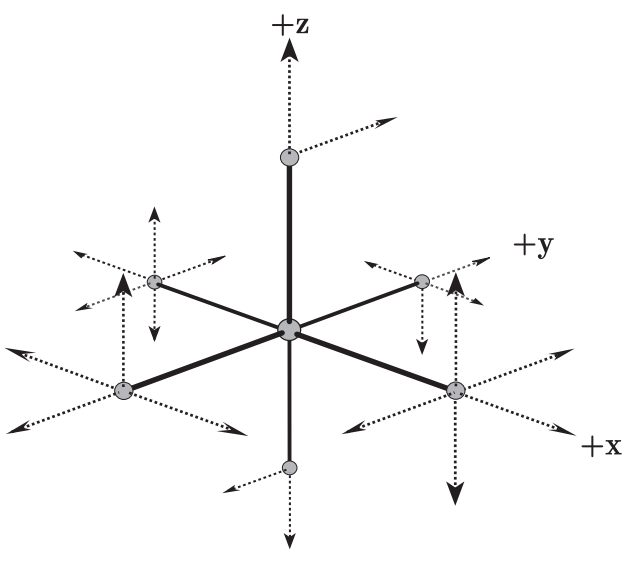

(S-C-P)

Figure 5. An illustration of the $(P-O-3)$ rule.

Figure 6. An illustration of the $(S-C-P)$ rule.

this rule is

$$
M_{\text {Rot }-\pi}=\left[\begin{array}{cccccc}
1 & 0 & 1 & 0 & 1 & 0 \\
0 & 1 & 0 & 1 & 0 & 1 \\
0 & 1 & 1 & 0 & 0 & 1 \\
1 & 0 & 0 & 1 & 1 & 0 \\
0 & 0 & 1 & 1 & 1 & 0 \\
1 & 1 & 0 & 0 & 0 & 1
\end{array}\right]
$$

\section{Exact enumeration results and analysis}

We now describe the enumeration and analyses of the 11 TSRW models constructed plane-byplane, in the manner described in the section 2.4. The rules were (3-3-C), $(S-C-3),(P-2-2)$, $(P-R-2),(P-3-3),(P-O-3),(P-P-P),(S-P-3),(P-P-3),(P-P-D)$ and $(S-C-P)$. These were 


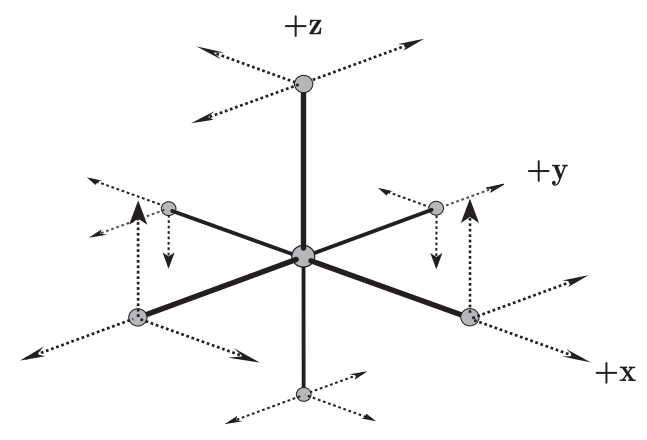

(P-R-2)

Figure 7. An illustration of the $(P-R-2)$ rule.

chosen from the original 38 models on which we performed short enumerations, not detailed here. We also considered the (Rot- $\pi$ ) rule, described above, to ensure the different possible symmetry combinations are covered. After the analysis of the 12 models we concentrated on the five models that were numerically best behaved and representative of the numerical behaviour found in the 12 models.

We began by enumerating the numbers of walks, $c_{n}$, and the total radius of gyration, $r_{n}$, where $\left\langle R_{g}^{2}\right\rangle_{n}=r_{n} / c_{n}$, for each of the 12 models listed above using a recursive back-tracking algorithm up to various maximum lengths, $n \leqslant N$, that depended on the model. (Later we also calculated the full moment of inertia for five of the models.) We chose to calculate and analyse the radius of gyration rather than the end-to-end distance since in two-dimensional studies the asymptotic analysis of the radius of gyration [13] has proven less affected by corrections-toscaling, as discussed in section 2 . The lengths of the enumerations depended on the effective connective constants of the models, and our enumerations ranged in length, $N$, from 18 to 29. The initial enumerations of the 12 models are given in appendix C. In particular, we have increased the length of the enumerations for the $(P-P-P)$ model from 23 [14] to 29 and the $(S-C-3)$ model from 18 [14] to 23 steps. As an example, the $(P-P-P)$ enumerations up to length 28 took approximately 150 CPU hours on a Digital Alphastation 500/266.

\subsection{Review of previous three-dimensional work}

Unrestricted SAWs, $(S-S-S)$, on the cubic lattice have been studied by both exact enumeration [16] and Monte Carlo techniques [23, 24]. For unrestricted SAWs scaling theory [1] predicts that the number of walks scales as

$$
c_{n} \sim B \mu^{n} n^{\gamma-1} \quad \text { as } n \rightarrow \infty
$$

so

$$
\sum_{n=0}^{\infty} c_{n} x^{n} \sim B^{\prime}(1-\mu x)^{-\gamma} \quad \text { as } \quad x \rightarrow \frac{1}{\mu^{-}}
$$

and that the total radius of gyration scales as

$$
r_{n} \sim D \mu^{n} n^{2 v+\gamma-1} \quad \text { as } n \rightarrow \infty
$$

so

$$
\sum_{n=0}^{\infty} r_{n} x^{n} \sim D^{\prime}(1-\mu x)^{-(2 v+\gamma)} \quad \text { as } \quad x \rightarrow \frac{1}{\mu^{-}}
$$


while the average radius of gyration scales as

$$
\left\langle R_{g}^{2}\right\rangle_{n} \sim A n^{2 v} \quad \text { as } n \rightarrow \infty
$$

so

$$
\sum_{n=0}^{\infty}\left\langle R_{g}^{2}\right\rangle_{n} x^{n} \sim A^{\prime}(1-x)^{-(2 v+1)} \quad \text { as } \quad x \rightarrow 1^{-} .
$$

An exact enumeration study [16] on the simple cubic lattice, of SAWs up to length 21 found

$$
\begin{aligned}
& \frac{1}{\mu}=0.213496(4) \\
& \gamma=1.161(1)
\end{aligned}
$$

and

$$
2 v=1.184(8)
$$

Various high-precision Monte Carlo studies using walks up to lengths $N=40000$ and $N=80000$ respectively have found $\gamma=1.1575(6)$ [24] and $2 v=1.1754(12)$ [23]. To make a fair comparison with our series analysis of the 12 TSRW we shall only use the exact enumeration results quoted above for the unrestricted SAW model.

Guttmann and Wallace [14] studied both the $(P-P-P)$ and $(S-C-3)$ model by exact enumeration, with walk lengths up to 23 and 18 respectively. They calculated the end-toend distance rather than the radius of gyration. They used a differential approximant analysis, and also various ratio analyses to study the models. The differential approximant analysis concluded that for the $(P-P-P)$ model

$$
\begin{aligned}
& \frac{1}{\mu}=0.3765(2) \\
& \gamma=1.24(20)
\end{aligned}
$$

and

$$
2 v=1.3(4)
$$

while for the $(S-C-3)$ model

$$
\begin{aligned}
& \frac{1}{\mu}=0.2883(2) \\
& \gamma=1.16(2)
\end{aligned}
$$

and

$$
2 v=1.19(5)
$$

The ratio methods gave more precise answers for the geometric-size exponents, $2 v=1.29(3)$ for the $(P-P-P)$ model, and $2 v=1.18(1)$ for the $(S-C-3)$ model. Note that, despite having similar numbers of terms as the unrestricted SAW enumeration, the analyses for these models give far less precise exponent estimates, and the series are far less well behaved under differential approximant analysis. Despite the large error bars on the differential approximant analyses, the further analyses via the ratio method led the authors to conjecture that the $(S-C-3)$ model is a member of the unrestricted SAW universality class while the $(P-P-P)$ model is part of a novel class, being a three-dimensional counterpart to the $2 \mathrm{D}$-spiral class $(P)$. They noted that both $(S-C-3)$ rule and unrestricted SAWs have a plane of reflection symmetry while the $(P-P-P)$ rule does not. They then concluded that this may be the microscopic criterion for the difference in the universality class, as it is in two dimensions. We note in passing that, while $(S-C-3)$ possesses a rotation-by- $\pi$ symmetry, the $(P-P-P)$ rule has no rotation symmetry (in contradiction to the claim made in [14]), so that could equally well be the microscopic criterion. 


\subsection{Differential approximant analysis of $c_{n}$ and $\left\langle R_{g}^{2}\right\rangle_{n}$ for $12 \mathrm{TSRW}$ models}

We first performed differential approximant analyses [25] on the number of walks, $c_{n}$ and the radius of gyration $\left\langle R_{g}^{2}\right\rangle_{n}$ for the 12 TSRW models. We used second-order inhomogeneous approximants that utilized all the available coefficients, and we varied the range of the order of the polynomials to suit the lengths of the series. We checked some of the analyses with first- and third-order approximants. In general, the first-order approximants were not as well behaved and the third-order approximants gave similar results to the second-order approximants, though they could not be used effectively due to the short length of many of the series.

Since we had no a priori estimates for the critical points of the various $c_{n}$ series, we arrived at our $\frac{1}{\mu}$ and $\gamma$ estimates by using unbiased approximants, and obtained error estimates from the spread (standard deviations) of the approximants. The estimates calculated were the mean values and the errors were two standard deviations.

We then considered the radius of gyration series, $\left\langle R_{g}^{2}\right\rangle_{n}$. Since the generating function of $\left\langle R_{g}^{2}\right\rangle_{n}$ should have a critical point equal to unity we used this as one measure of the convergence of that series. Many of the $\left\langle R_{g}^{2}\right\rangle_{n}$ series were poorly converged compared with the unrestricted SAW series. Most also had a relatively wide spread of approximants. We found that using 'biased approximants', as per [25], yielded very poor results. Instead we arrived at a biased exponent estimate from the unbiased approximants in the following way: we took a large range of (unbiased) approximants, made a linear fit on the central section of the approximants and then extrapolated back to a critical point of $x=1$. The various estimates and associated errors we calculated were taken from this linear fit. Firstly, we calculated the mean critical point and exponent of the approximants on which the linear fit was taken, which we denote $\bar{x}_{c}$ and $2 \bar{v}$ respectively (errors are two standard deviations). Secondly, we calculated the estimate from linear biasing itself, $2 v_{\mathrm{LB}}$ (error quoted is the error from the linear regression). The final estimate, $2 v_{\text {final }}\left(\Delta_{\text {stat }}\right)\left(\Delta_{\text {sys }}\right)$ is the same as the biased value, $2 v_{\mathrm{LB}}$, but quoted with two errors: $\Delta_{\text {stat }}$ is the statistical error from the linear regression; while $\Delta_{\text {sys }}$ is a measure of the systematic error in biasing the approximants back to unity-it is equal to the difference between the mean exponent value and biased value, $\left|2 v_{\mathrm{LB}}-2 \bar{v}\right|$. Using $\Delta_{\text {sys }}$ for our estimate of systematic error may be considered rather conservative, but this estimate has proved to be a useful measure of systematic error in other SAW problems, such as polymer adsorption [26]. A typical differential approximant spread for the $\left\langle R_{g}^{2}\right\rangle_{n}$ series is shown in figure 8, with illustrations of the various estimates and associated errors we calculated given.

The results from the differential approximant analysis are given in table 3 . Considering the entropic exponent $\gamma$ estimates in table 3, we find that the SAW value of 1.161 falls within the respective errors estimated for all the models. This would suggest one reasonable conclusion to be that all the models are in the same universality class as unrestricted SAWs. The $\left\langle R_{g}^{2}\right\rangle_{n}$ approximants, by contrast, seem to indicate that the models fall into possibly three different universality classes:

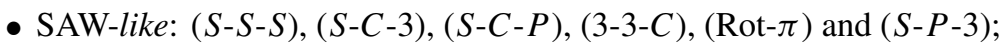

- New: $(P-3-3),(P-O-3)$ and $(P-2-2)$;

- 3D-spiral: $(P-P-P)$ and $(P-R-2)$.

We were unable to classify the $(P-P-3)$ rule because of the size of the associated error bars on the exponent estimates; however, it seems to lie in either the apparent 3D-spiral class or the new class, though it may, of course, form another class again. Though the results from the analysis of the data are too poor to draw any reasonable conclusions we have included the $(P-P-D)$ data because of the symmetries of the walk rule and its winding number.

The spread of $\left\langle R_{g}^{2}\right\rangle_{n}$ approximants for several representative rules is shown in figure 9. This figure clearly shows what appears to be three separate bands of approximants representing 


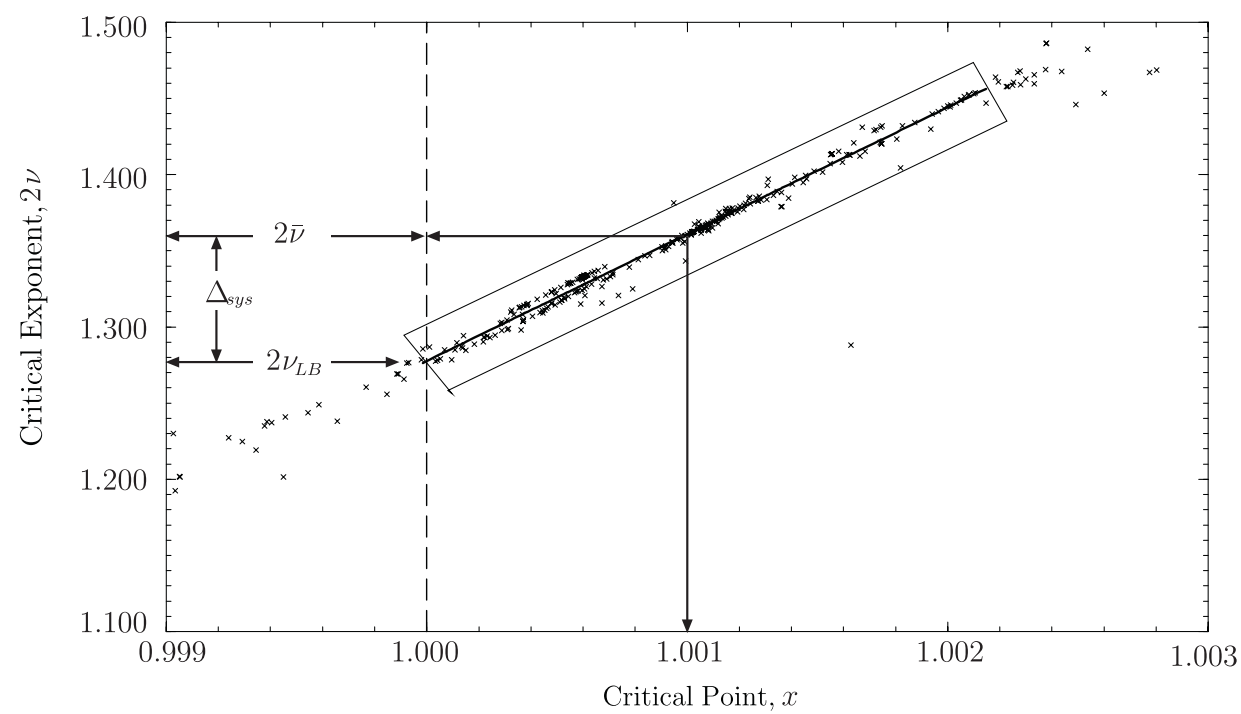

Figure 8. The differential approximant spread for the radius of gyration exponent, $2 v$, for the $(P-P-P)$ model. The box indicates the area over which an average was taken. A linear fit is shown which gives our 'biased' estimate, $2 v_{\mathrm{LB}}$, from the point where the fit has an intercept with the dashed $x=1$ vertical line. The arrows indicate the means of the boxed region's critical point, $\bar{x}_{c}$, and exponent, $2 \bar{v}$. The difference between the boxed mean, $2 \bar{v}$, and the biased estimate, $2 v_{\mathrm{LB}}$, of the critical exponent gives us an estimate of systematic error, $\Delta_{\text {sys }}$ : in other walk problems this has usually proved to be conservative, though not always.

Table 3. Exponent estimates from differential approximant analysis. There are two sets of results for the $(P-P-P)$ model, corresponding to second order and third order approximants. We include, for completeness, two sets of SAW $(S-S-S)$ values, both obtained from exact enumeration data, one using our analysis method and one quoted from previous work.

\begin{tabular}{lllllll}
\hline Rule & $1 / \mu$ & $\gamma$ & $\bar{x}_{c}$ & $2 \bar{v}$ & $2 \nu_{\mathrm{LB}}$ & $2 \nu_{\text {final }}$ \\
\hline$(S-C-3)$ & $0.2884(1)$ & $1.16(2)$ & $1.0002(4)$ & $1.20(2)$ & $1.19(1)$ & $1.19(1)(1)$ \\
$(3-3-C)$ & $0.2904(2)$ & $1.16(2)$ & $1.0005(6)$ & $1.22(3)$ & $1.19(1)$ & $1.19(1)(3)$ \\
$(S-P-C)$ & $0.3046(2)$ & $1.17(2)$ & $1.0002(7)$ & $1.21(3)$ & $1.20(1)$ & $1.20(1)(1)$ \\
$(S-P-3)$ & $0.26985(4)$ & $1.169(6)$ & $1.0003(3)$ & $1.22(2)$ & $1.21(1)$ & $1.21(1)(1)$ \\
$($ Rot- $\pi)$ & $0.3575(4)$ & $1.17(4)$ & $0.9997(14)$ & $1.19(7)$ & $1.20(1)$ & $1.20(1)(1)$ \\
$(P-3-O)$ & $0.4078(2)$ & $1.18(3)$ & $0.9999(4)$ & $1.22(2)$ & $1.226(6)$ & $1.23(1)(1)$ \\
$(P-3-3)$ & $0.30558(8)$ & $1.173(14)$ & $1.0002(2)$ & $1.23(1)$ & $1.220(5)$ & $1.22(1)(1)$ \\
$(P-2-2)$ & $0.3442(1)$ & $1.18(1)$ & $1.0003(3)$ & $1.25(2)$ & $1.23(1)$ & $1.23(1)(2)$ \\
$(P-P-3)$ & $0.33167(7)$ & $1.176(8)$ & $1.0008(2)$ & $1.30(2)$ & $1.25(2)$ & $1.25(2)(5)$ \\
$(P-P-D)$ & $0.3586(4)$ & $1.17(4)$ & $1.004(2)$ & $1.50(8)$ & $1.33(11)$ & $1.33(11)(17)$ \\
$(P-2-R)$ & $0.3739(2)$ & $1.19(2)$ & $1.0011(6)$ & $1.36(3)$ & $1.30(2)$ & $1.30(2)(6)$ \\
$(P-P-P)$ 2nd & $0.3757(1)$ & $1.18(2)$ & $1.001(1)$ & $1.37(6)$ & $1.29(2)$ & $1.29(2)(8)$ \\
$(P-P-P)$ 3rd & $0.3757(1)$ & $1.17(4)$ & $1.001(1)$ & $1.36(10)$ & $1.27(2)$ & $1.27(2)(9)$ \\
SAW-here & $0.213497(10)$ & $1.162(2)$ & $1.0002(2)$ & $1.20(1)$ & $1.192(5)$ & $1.19(1)(1)$ \\
SAW-there & $0.213496(4)$ & $1.161(2)$ & - & - & - & $1.184(6)$ \\
\hline
\end{tabular}

three separate universality classes. Our results in this section generally concur with those of Guttmann and Wallace [14] for the $(S-C-3)$ and $(P-P-P)$ models. On the other hand, it is difficult to be confident in the conclusions of these results due to the relatively large systematic errors in the $v$ estimates that arise from the biasing procedure. It is possible that the conclusion 


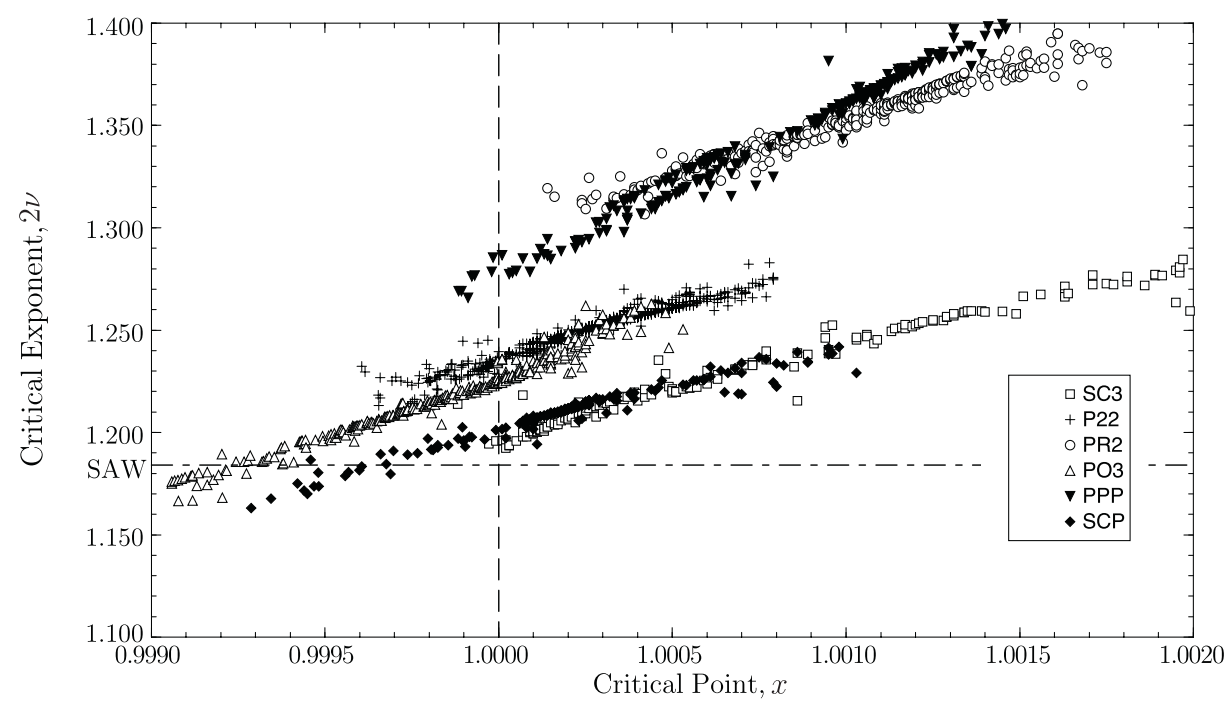

Figure 9. A plot of $\left\langle R_{g}^{2}\right\rangle_{n}$ differential approximants for the exponent $2 v$ for several representative models. Notice the three distinct bands of approximants, each one made up from the approximants for several models. These bands seem to indicate that there are three universality classes.

that there is more than one universality class is a manifestation of corrections-to-scaling: the series being too short for the method of differential approximants to work effectively.

Assuming for the moment that the conclusions of the $\left\langle R_{g}^{2}\right\rangle_{n}$ analysis are true, our next task was to attempt to establish the microscopic criteria that classify rules according to these apparent universality classes. We attempted this using the criteria that have proved useful in two dimensions. Given that all the rules are symmetric-balanced the various rule symmetries and the turning numbers were the candidates considered. The symmetries and turning numbers of these rules along with the maximum exact enumeration lengths are shown in table 4. All rules except $(\operatorname{Rot}-\pi)$ were flip symmetric. Note that the unrestricted SAW model, $(S-S-S)$, has all the symmetries listed in table 4 . Also, any rule that is symmetric under rotations by $\frac{\pi}{2}$ must be symmetric under rotations by $\pi$ and also, as mentioned in section 2.3.2, must possess a reflection symmetry. Hence, the unrestricted SAW rule $(S-S-S)$ is such a rule.

One can see immediately that all the rules in the new class, in the $3 \mathrm{D}$-spiral class, and some in the SAW-like class do not have any rotation or reflection symmetries. Hence, these symmetries cannot be used to classify models into the apparent classes. In particular, reflection symmetry does not delineate the SAW class from the others. Hence, a classification according to symmetries of the rules is not forthcoming. A classification according to turning number would seem to be more successful (increasing turning number giving new classes at particular values, 20 and 36 perhaps). Here also there are problems: both the $(S-P-3)$ and $(P-O-3)$ rules have turning number 20 but seem to be in different universality classes and it is unclear in which class the model $(P-P-3)$ lies despite having the same turning number as the model $(P-R-2)$. These difficulties with an attempted microscopic classification and the consistency of the values of $\gamma$ with the SAW value across all the rule models led us to examine the scaling behaviour of the models both using different quantities and with different analysis techniques in an attempt to find a consistent answer to this conflicting information.

We chose to examine five models representative of the apparent universality classes: $(P$ $P-P)$ and $(P-R-2)$ for the $3 \mathrm{D}$-spiral class, $(P-2-2)$ and $(P-O-3)$ for the new class, and $(S-C-P)$ 
Table 4. Symmetries of the TSRW models examined. They are grouped according to the initial classification made from differential approximant analysis of the exact enumerations of the radius of gyration. The maximum length of the enumerations $N$ is also given.

\begin{tabular}{lllllll}
\hline Class & Rule & $N$ & $\begin{array}{l}\text { Rotation } \\
\text { by } \frac{\pi}{2}\end{array}$ & $\begin{array}{l}\text { Rotation } \\
\text { Rot- } \pi\end{array}$ & $\begin{array}{l}\text { Any } \\
\text { reflection }\end{array}$ & $\begin{array}{l}\text { Turning } \\
\text { number }\end{array}$ \\
\hline SAW-like & $(S-S-S)$ & 21 & $\mathrm{y}$ & $\mathrm{y}$ & $\mathrm{y}$ & 0 \\
& $(S-C-3)$ & 23 & $\mathrm{n}$ & $\mathrm{y}$ & $\mathrm{y}$ & 4 \\
& $(3-3-C)$ & 20 & $\mathrm{n}$ & $\mathrm{n}$ & $\mathrm{n}$ & 8 \\
& $(S-C-P)$ & 22 & $\mathrm{n}$ & $\mathrm{y}$ & $\mathrm{y}$ & 16 \\
\multirow{4}{*}{ New } & $($ Rot $-\pi)$ & 22 & $\mathrm{n}$ & $\mathrm{y}$ & $\mathrm{n}$ & 16 \\
& $(S-P-3)$ & 18 & $\mathrm{n}$ & $\mathrm{n}$ & $\mathrm{n}$ & 20 \\
& $(P-O-3)$ & 28 & $\mathrm{n}$ & $\mathrm{n}$ & $\mathrm{n}$ & 20 \\
Undetermined & $(P-3-3)$ & 24 & $\mathrm{n}$ & $\mathrm{n}$ & $\mathrm{n}$ & 24 \\
& $(P-2-2)$ & 24 & $\mathrm{n}$ & $\mathrm{n}$ & $\mathrm{n}$ & 24 \\
3D-spiral & $(P-P)$ & 22 & $\mathrm{n}$ & $\mathrm{n}$ & $\mathrm{y}$ & 32 \\
& $(P-P-2)$ & 25 & $\mathrm{n}$ & $\mathrm{n}$ & $\mathrm{n}$ & 36 \\
& $(P-P-P)$ & 29 & $\mathrm{n}$ & $\mathrm{n}$ & $\mathrm{n}$ & 36 \\
& & & & $\mathrm{n}$ & $\mathrm{n}$ & 48 \\
\hline
\end{tabular}

representing the SAW class. We first considered further analysis of the $c_{n}$ series that attempted to take account of non-standard scaling forms such as occurs in the two-dimensional spiral class $(P)$, and secondly we analysed the mean moment of inertia tensor, $\langle\boldsymbol{I}\rangle_{n}$, to search for exponent anisotropy as occurs in the two-dimensional anisotropic spiral class $(A)$.

\subsection{Further analysis of $c_{n}$ for five TSRW models}

As stated above, we first expected that the number of walks, $c_{n}$, behaved according to equation (3.1) for each of the rule models: the differential approximant analysis described above fits to this form (with implicit corrections). However, different scaling forms have been found in some two-dimensional walk models. Two-dimensional spiral walks [5] and walks of the ASSAW class [11] have scaling forms that include $\mathrm{e}^{\sqrt{n}}$ factors (see table 1). This additional factor present in the spiral walks' partition function scaling is mathematically related to the scaling of partitions of integers [5]; in three dimensions it may be possible that there will be plane-partition-like terms which scale as $[27,28]$

$$
p_{n} \sim C n^{-25 / 36} \mathrm{e}^{a_{P P} n^{2 / 3}} \quad \text { as } n \rightarrow \infty .
$$

We examined the possibility of corrections to the scaling form of the sort found in twodimensional spirals and also those found in plane partitions. Differential approximant analysis (at least without significant modification) is unsuited to the study of such scaling forms, since the new factors imply that the generating function has an essential singularity. Of course, if such factors are present then the previous differential approximant analysis would have been inappropriate. With this in mind we attempted to make a direct fit of the data to the following forms:

$$
\begin{array}{ll}
c_{n} \sim C \mu^{n} n^{\gamma-1} \quad \text { as } \quad n \rightarrow \infty & \\
c_{n} \sim C \mu^{n} n^{\gamma-1} \exp (\alpha \sqrt{n}) \quad \text { as } \quad n \rightarrow \infty
\end{array}
$$

and

$$
c_{n} \sim C \mu^{n} n^{\gamma-1} \exp \left(\alpha n^{2 / 3}\right) \quad \text { as } \quad n \rightarrow \infty .
$$


More precisely, we made successive fits using the series terms $n, n-2, n-4$ and $n-6$, as necessary, to each of the forms (which (linearly) contain 3 or 4 constants to ascertain)

$$
\begin{aligned}
& \log c_{n}=a_{1}+n \log \mu+(\gamma-1) \log n \\
& \log c_{n}=a_{1}+n \log \mu+(\gamma-1) \log n+a_{2} \sqrt{n}
\end{aligned}
$$

and

$$
\log c_{n}=a_{1}+n \log \mu+(\gamma-1) \log n+a_{2} n^{2 / 3}
$$

exactly. We made these fits with either $\gamma$ free, or fixed at the SAW value (in the second case we used one less term at each stage). We did not attempt to consider multiplicative logarithmic corrections, as well as those above, since such forms are too difficult to fit with all but the longest series. The type of analysis described above is more refined than ratio analysis and has been used to great effect in SAW problems when considering corrections-to-scaling [29].

We illustrate the results of this fitting procedure in detail for the $(P-P-P)$ walks in appendix D.2. To summarize, after examining tables D1 and D2, one can see that if corrections of the form $\mathrm{e}^{\sqrt{n}}$ or $\mathrm{e}^{n^{2 / 3}}$ are introduced, then they have coefficients very close to zero. This implies that any effect of these terms is quite negligible, and indeed that they are probably not present. We have found similar results for the other four walk models considered. This gives us confidence that the differential approximant analysis of $c_{n}$ is giving reliable information. Hence, given that $\mathrm{e}^{\sqrt{n}}$ or $\mathrm{e}^{n^{2 / 3}}$ corrections seem not to be present in the scaling form for $c_{n}$, our original conclusion from the differential approximant analysis then stands: namely, that all the rules, including $(S-S-S)$, have the same value of the $\gamma$ exponent within error, being that of the SAW universality class (around 1.16). If the walks scale with subtly different exponents or multiplicative logs, the series at hand are too short to allow an investigation of these possibilities.

\subsection{Analysis of the inertia tensor for five TSRW models}

In our initial enumerations of the 12 models (as well as our very initial enumerations-not described here - of the 38 models of section 2.4) we enumerated the radius of gyration so as to measure the scaling of the average size of walks in the various models. To gain a finer view of the scaling of the geometric size we calculated the full moment of inertia tensor for the five models we designated for more intense study. This allowed us to look for any scaling anisotropy and to consider the eigenvalues of this matrix in addition to the radius of gyration.

The eigenvectors of the moment of inertia matrix correspond to the natural coordinate axes in which the ('average') walks scale, and the eigenvalues to the radius of gyration in those directions. For example, in two dimensions the three-choice walk model [12] has a mean moment of inertia tensor with eigenvectors $\{[1,1],[1,-1]\}$, which correspond to the preferred and transverse directions. The eigenvalues for any three-dimensional TSRW model's moment of inertia matrix are expected to scale as

$$
\lambda_{j}(n) \sim A_{j} n^{2 v_{j}} \quad j=1,2,3 \quad \text { as } \quad n \rightarrow \infty
$$

where the values of $v_{j}$ and $A_{j}$ may or may not be independent of direction $j$. Hence, there are two types of anisotropy: scaling anisotropy where different eigenvalues scale with different exponents $\left(v_{i} \neq v_{j}\right)$, and a milder anisotropy where only the constants $A_{j}$ differ. The moment of inertia tensor, $\boldsymbol{I}$, for a walk configuration $\varphi_{n}$ of $n$ steps, defined by the positions of its $n+1$ monomers (sites) as the set $\left\{\vec{r}_{i}=\left(x_{i}, y_{i}, z_{i}\right) ; i=0, \ldots, n\right\}$, is given by

$$
\boldsymbol{I}\left(\varphi_{n}\right)=\frac{1}{n+1} \sum_{i=0}^{n}\left(r_{i}^{2} \mathbf{1}-\vec{r}_{i} \vec{r}_{i}\right)
$$


Table 5. Estimates of $2 v$ from the differential approximant analysis of the eigenvalues of the mean moment of inertia tensor computed about the centre of mass. Estimates of the critical points $x_{i}$ of the associated generating functions are included to show quality of convergence. $(P-P-P)$ has two eigenvalues, the larger having multiplicity 2 . SAW has only one eigenvalue and the estimate here comes from [16]. We also note here that for the $(P-R-2)$ model although $\lambda_{3} \geqslant \lambda_{2}$ we estimate $v_{2} \geqslant v_{3}$. This implies that corrections to scaling are masking that $v_{2}=v_{3}$.

\begin{tabular}{|c|c|c|c|c|c|c|}
\hline \multirow[b]{2}{*}{ Rule } & \multicolumn{2}{|c|}{$\lambda_{1}$} & \multicolumn{2}{|c|}{$\lambda_{2}$} & \multicolumn{2}{|c|}{$\lambda_{3}$} \\
\hline & $x_{1}$ & $2 v_{1}$ & $x_{2}$ & $2 v_{2}$ & $x_{3}$ & $2 v_{3}$ \\
\hline SAW & 1 & $1.184(6)$ & - & - & - & - \\
\hline$(S-C-P)$ & $0.9995(10)$ & $1.18(2)(2)$ & $1.0004(6)$ & $1.21(1)(1)$ & $1.0007(7)$ & $1.21(2)(3)$ \\
\hline$(P-O-3)$ & $0.9997(4)$ & $1.20(1)(2)$ & $0.9999(8)$ & $1.23(2)(1)$ & $1.0002(5)$ & $1.23(1)(2)$ \\
\hline$(P-2-2)$ & $1.0002(4)$ & $1.22(1)(1)$ & $1.0003(5)$ & $1.24(1)(1)$ & $1.0002(4)$ & $1.24(1)(2)$ \\
\hline$(P-R-2)$ & $1.0005(14)$ & $1.24(1)(4)$ & $1.0015(7)$ & $1.33(4)(7)$ & $1.0011(6)$ & $1.31(3)(5)$ \\
\hline$(P-P-P) 2 \mathrm{nd}$ & $0.9997(16)$ & $1.22(3)(2)$ & $1.0058(20)$ & $1.26(12)(45)$ & - & - \\
\hline$(P-P-P) 3 \mathrm{rd}$ & $0.9991(24)$ & $1.22(3)(7)$ & $1.0021(26)$ & $1.29(2)(16)$ & - & - \\
\hline
\end{tabular}

where we have used dyadic notation, and $\mathbf{1}$ is the identity tensor. Explicitly, this gives

$$
\begin{aligned}
\boldsymbol{I}\left(\varphi_{n}\right) & =\sum_{i=0}^{n}\left(\left[\begin{array}{ccc}
r_{i}^{2} & 0 & 0 \\
0 & r_{i}^{2} & 0 \\
0 & 0 & r_{i}^{2}
\end{array}\right]-\left[\begin{array}{ccc}
x_{i} x_{i} & x_{i} y_{i} & x_{i} z_{i} \\
y_{i} x_{i} & y_{i} y_{i} & y_{i} z_{i} \\
z_{i} x_{i} & z_{i} y_{i} & z_{i} z_{i}
\end{array}\right]\right) \\
& =\sum_{i=0}^{N}\left[\begin{array}{ccc}
y_{i}^{2}+z_{i}^{2} & -x_{i} y_{i} & -x_{i} z_{i} \\
-x_{i} y_{i} & x_{i}^{2}+z_{i}^{2} & -y_{i} z_{i} \\
-x_{i} z_{i} & -y_{i} z_{i} & x_{i}^{2}+y_{i}^{2}
\end{array}\right] .
\end{aligned}
$$

In our enumerations we computed the expectation of the moment of inertia tensor averaging over all walks $\varphi_{n}$ of length $n$, that is,

$$
\langle\boldsymbol{I}\rangle_{n}=\frac{1}{c_{n}} \sum_{\varphi_{n}} \boldsymbol{I}\left(\varphi_{n}\right)
$$

where the sum is over all walks, $\varphi_{n}$, of length $n$. The value of the moment of inertia depends on the origin of the coordinate system. Now, the trace of the moment of inertia tensor yields

$$
\operatorname{Tr}\left(\langle\boldsymbol{I}\rangle_{n}\right)=\frac{1}{c_{n}} \sum\left(\frac{2}{n+1} \sum_{i=0}^{n}\left(x_{i}^{2}+y_{i}^{2}+z_{i}^{2}\right)\right)
$$

which is equal to twice the mean square distance of a monomer to the endpoint if $I$ is computed about one endpoint of the walks, or $2\left\langle R_{g}^{2}\right\rangle$ if $\boldsymbol{I}$ is computed about the centre of mass. So, to expand on our radius of gyration enumerations, we enumerated the centre of mass $\frac{1}{n+1} \sum_{j=0}^{n} \vec{r}_{j}$ for each walk and then the components of the moment of inertia matrix about the centre of mass. The enumerations of the mean moment of inertia tensor computed about the centre of mass for our five models are given in appendix C.2.

In a similar manner to the analysis of the $\left\langle R_{g}^{2}\right\rangle_{n}$ data, we used second-order inhomogeneous differential approximants to analyse the scaling of the eigenvalues of the mean moment of inertia tensor computed about the centre of mass, which we denote as $\lambda_{1}(n), \lambda_{2}(n)$ and $\lambda_{3}(n)$ in ascending order of size. Note that since the trace of a matrix is the sum of its eigenvalues the scaling of the mean square monomer-to-end distance and the radius of gyration are dominated by the scaling of the largest eigenvalue. The results of this analysis are given in table 5 .

These results for each of the five models indicate that the smallest eigenvalue, $\lambda_{1}(n)$, apparently scales with an exponent that is smaller than the exponent associated with the two larger eigenvalues. These two larger eigenvalues seem to scale with approximately the same 
exponent, that is $v_{2}=v_{3}$. The value obtained for this exponent is the same as the one obtained from the analysis of the radius of gyration. This anisotropic scaling implies that the typical walk looks like a flattened ball, and that the ball becomes flatter as the walk length increases. It is interesting to note that the degree of anisotropy in the exponents seems to be of the order of $5 \%$, in contrast to $50 \%$ found in the two-dimensional ASSAW (and DW) models. If it were true that this anisotropy were real it would indeed be curiously subtle, and unusual, for threedimensional critical phenomena, as far as we are aware. Intriguingly, on closer examination of the results we find that the smallest eigenvalue is also typically the best converged, and it is (with the exception of the $(P-R-2)$ model), well converged to a SAW-like value close to 1.20 with errors that encompass the best series estimate of the unrestricted SAW model of 1.184(6). So the best converged (the biased shift in the exponent is about the same size as the statistical spread of approximants and is about 0.01) differential approximant analysis is for the smallest eigenvalue: one might expect naively that the smallest eigenvalue is affected most by corrections-to-scaling and so behaves the worst under scaling analysis. Moreover, the analyses of the largest eigenvalues have such large systematic errors that the estimate-ranges often encompass (sometimes just so) the SAW value of 1.184(6). It is then advantageous to attempt to analyse these eigenvalue scalings again with other techniques.

Because of these unusual results we then analysed the series data again, making allowances for the existence of analytic and non-analytic corrections-to-scaling by fitting to an assumed scaling form in much the same manner as [29], and as we did for the further scaling of $c_{n}$ in section 3.3. In particular, we examined the cases of analytic corrections (to order $\frac{1}{n^{2}}$ ) and non-analytic corrections of the form $\frac{1}{\sqrt{n}}$ as reasonable guesses, assuming some compatibility with previous SAW work: that is, we considered

$$
\lambda(n) \sim A n^{2 v}\left(1+\frac{c_{1}}{n}+\frac{c_{2}}{n^{2}}+\mathrm{O}\left(\frac{1}{n^{3}}\right)\right) \quad \text { as } n \rightarrow \infty
$$

and

$$
\lambda(n) \sim A n^{2 v}\left(1+\frac{c_{2}}{\sqrt{n}}+\frac{c_{1}}{n}+\mathrm{O}\left(\frac{1}{n^{3 / 2}}\right)\right) \quad \text { as } n \rightarrow \infty .
$$

We did not fit directly to the above scaling form (since we did not have conjectured values of $2 v$ ); rather we fitted to the term-by-term logarithm of the series

$$
\log (\lambda(n))=a_{1}+2 v \log (n)+\frac{a_{2}}{n}+\frac{a_{3}}{n^{2}}+\mathrm{O}\left(\frac{1}{n^{3}}\right)
$$

and

$$
\log (\lambda(n))=a_{1}+2 v \log (n)+\frac{a_{3}}{\sqrt{n}}+\frac{a_{2}}{n}+\mathrm{O}\left(\frac{1}{n^{3 / 2}}\right) .
$$

The (linear) fits were then examined on the basis of the stability of the coefficients. For each of these two forms above the exponent $2 v$ was either allowed to be free, fixed at a SAW-like value of 1.19, or fixed to the apparent differential approximant calculated estimate obtained previously (1.29 for the 3D-spiral models and 1.23 for the new class).

We illustrate this analysis in more detail for $(P-P-P)$ walks.

3.4.1. Exact fitting to corrections-to-scaling for $(P-P-P)$ model. For the $(P-P-P)$ model the mean inertia tensor takes the form

$$
\langle\boldsymbol{I}\rangle_{(P P P)}=\left[\begin{array}{ccc}
a & b & -b \\
b & a & b \\
-b & b & a
\end{array}\right]
$$




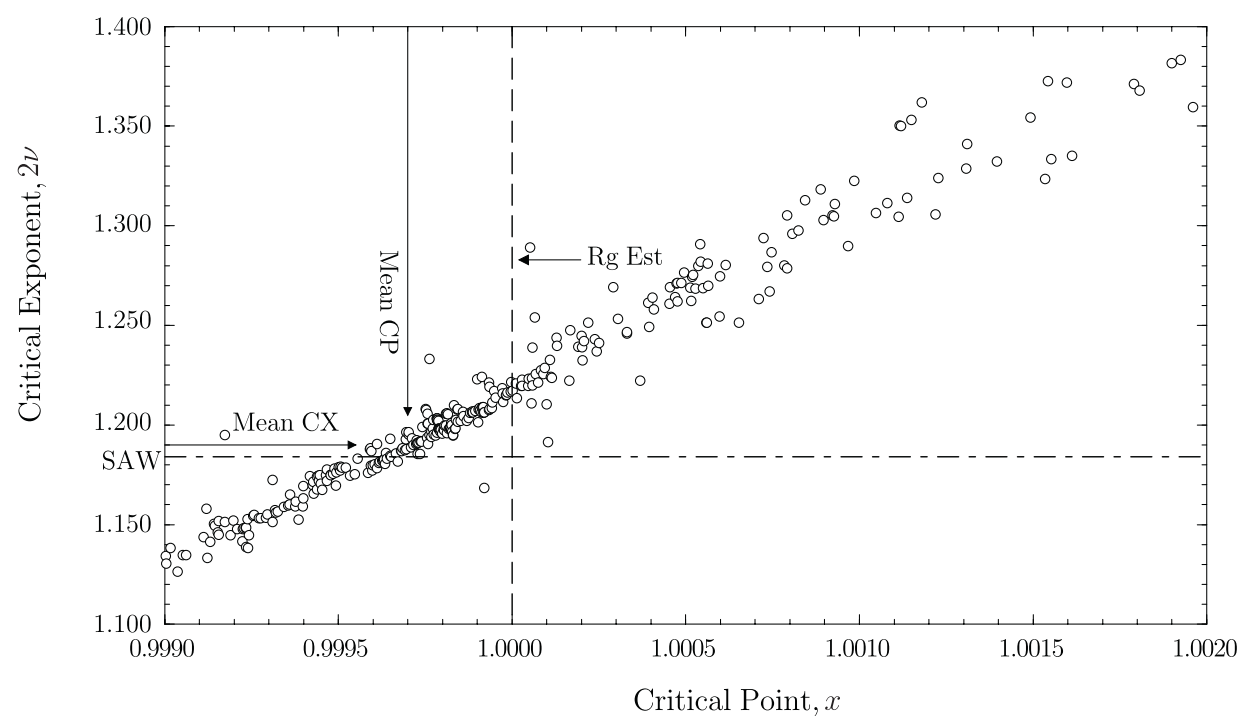

Figure 10. A plot of the $\lambda_{1}$ (smallest eigenvalue of the moment of inertia tensor) differential approximants for the $(P-P-P)$, which estimate the exponent $2 v$. The mean value of the critical points and exponents of these approximants is indicated. The vertical dashed line is simply the $x=1$ (correct) critical point, while the horizontal dashed line marks the SAW value of the exponent $2 v$. The estimate of the exponent from the radius of gyration analysis, $\mathrm{Rg}$ Est, is also marked.

and such a matrix has the following eigenvalues, $\lambda_{j}$, and eigenvectors, $\vec{\phi}_{j}$ :

$$
\lambda_{1}=a-2 b \quad \text { and } \quad\left\{\vec{\phi}_{1}=[1,-1,1]\right\}
$$

and

$$
\lambda_{2}=a+b \quad \text { and } \quad\left\{\vec{\phi}_{2}=[-1,0,1], \vec{\phi}_{3}=[1,1,0]\right\} .
$$

The first interesting feature to notice is that this rule is not automatically isotropic, unlike the 2D-spiral $(P)$ class model.

Differential approximant analysis on the two eigenvalues of the $(P-P-P)$ model yielded (see table 5 and figure 10):

$$
\begin{aligned}
& \lambda_{1} \sim A_{1} n^{2 v_{1}} \quad \text { as } n \rightarrow \infty \quad \text { with } 2 v_{1} \approx 1.22(5) \\
& \lambda_{2}=\lambda_{3} \sim A_{3} n^{2 \nu_{3}} \quad \text { as } n \rightarrow \infty \quad \text { with } 2 \nu_{3} \approx 1.29(18)
\end{aligned}
$$

where the errors quoted here are simply the sum of the statistical and systematic errors. The central estimates of the exponents imply that $(P-P-P)$ walks scale anisotropically, and that the typical walk is shaped like a flattened ball, shorter in the $[1,-1,1]$ direction, with a preferred plane normal to this. Since one eigenvalue apparently scales with a smaller exponent the ball becomes flatter as the walks become longer. Since the radius of gyration is the sum of the eigenvalues (up to a constant), the scaling of $\left\langle R_{g}^{2}\right\rangle_{n}$ is dominated by the scaling of the largest and less well-converged eigenvalues; this translates into the relatively poor convergence of the $\left\langle R_{g}^{2}\right\rangle_{n}$ series data for this model. On the other hand, both estimates' ranges include the SAW value of 1.19. So we might conclude that it is simply the case that the larger eigenvalues are afflicted with large corrections-to-scaling.

As mentioned above, it would be an unusual result if the walks scale as three-dimensional SAWs in one direction, and differently in the other two. To explore this result further we tried to fit the eigenvalues directly to the scaling forms given by equations (3.29) and (3.30), in much 
the same way as [29], to examine the possible corrections-to-scaling. The results of these fits are given in appendix D.2. In summary, the smallest eigenvalue is fitted best with standard $\frac{1}{n}$ corrections with a dominant exponent close to the unrestricted SAW value, in agreement with the differential approximant analysis. However, the larger eigenvalue can also be fitted with an exponent of $2 v=1.19$, which is close to the unrestricted SAW value, if $\frac{1}{\sqrt{n}}$ corrections (as in (3.30)) are added, and moreover, this is arguably the best fit of six attempted for the larger eigenvalue. Hence one can argue sensibly from these fits that a SAW-like exponent is not only consistent with, but rather the best value (of those tried) for all the eigenvalues of the $(P-P-P)$ model. See appendix D.2 for further details.

Similar results have been obtained for the other four models: $(P-O-3),(P-R-2),(S-C-P)$ and $(P-2-2)$. In particular, we see that the fitted values for $v$ drop with increasing $n$ with either $\frac{1}{n}$ or $\frac{1}{\sqrt{n}}$ corrections, suggesting that the differential approximant estimates (particularly for the larger eigenvalues) are too high.

We therefore can conclude from the above analyses that strong corrections-to-scaling occur in the scaling of largest eigenvalues of the moment of inertia (and hence in the radius of gyration) of the models in the apparent new universality class and also those in the 3D-spiral class. Furthermore, by including such corrections into the analysis the enumeration data is consistent with there being only one scaling exponent, namely that of the unrestricted SAW.

\section{Discussion}

We now review the various analyses and conflicting conclusions made from those above analyses to come to some global conclusions. In the TSRW models we have examined, the radius of gyration data confirms an earlier suggestion [14] of a three-dimensional spiral universality class. Our data also supports the new suggestion that there is at least one other novel class. Poor convergence of the series analyses, based upon differential approximants, makes this conclusion contentious however. On the other hand, examination of the number of walks, $c_{n}$, again using differential approximants, seems to indicate that all the models considered have approximately the same value (within error) of their entropic exponent $\gamma$ and, further, that the scaling of $c_{n}$ does not contain $\mathrm{e}^{\sqrt{n}}$ or $\mathrm{e}^{n^{2 / 3}}$ factors that may invalidate the differential approximant analyses. Moreover, the symmetries of the walk rules offer little insight into the differences in their $\left\langle R_{g}^{2}\right\rangle_{n}$ scaling, in distinction to two dimensions. The turning number of the rule, which we have defined here, does seem to be a better candidate as a microscopic criterion to differentiate the scaling behaviours of the TSRW models, though this too is problematic. Our examination of the scaling of the eigenvalues of the inertia tensor for five of the models indicates that the smallest eigenvalue is the best behaved numerically and, further, that it scales with an exponent quite close to that of the SAW universality class. While the analyses of the larger eigenvalues seem to be hampered by relatively larger corrections-to-scaling, they reflect the analysis of the radius of gyration which gives an exponent central-estimate larger than the SAW value. However, further analysis of the larger eigenvalues are also consistent with lower SAW-like values of the exponents.

The above facts leave us with a couple of alternate and reasonable conclusions. Of course, there are other possibilities given the moderate size of some of the errors on our analyses but the following are the most likely, consistent, and simplest conclusions. Either

(1) all the TSRW models in three dimensions we examined (and probably all symmetricmixing rules) are members of the unrestricted SAW universality class with different corrections-to-scaling (categorized maybe by the turning number) or

(2) the walk rules split into at least three universality classes with different values of the 
exponent $v$. The non-SAW-like classes have anisotropic exponents which are only slightly anisotropic (of the order of 5\%). Moreover, all classes have one exponent (the smallest) that takes on a value very close to the 3D-SAW one.

Also, we conclude that the exponent $\gamma$ for any of the TSRW models examined (and presumably all symmetric-mixing models) probably takes on the SAW value (around 1.16) or a value very close to it.

Now, given our further analysis of the moment of inertia eigenvalues the weight of evidence favours the first conclusion, we believe. In any event it would be advantageous indeed to have confirmation of some of these results using Monte Carlo simulations.

\section{Acknowledgments}

ALO is grateful to the Australian Research Council for financial support and AR thanks the University of Melbourne for an Australian Postgraduate Award. We thank A J Guttmann and W Orrick for carefully reading the manuscript and making several useful suggestions.

\section{Appendix A. Square lattice balanced and reverse-balanced walk rules}

Starting with the unrestricted SAW rule in two dimensions and deleting possible moves, whilst imposing the balance and reverse-balance condition, one arrives at all the interesting rules first: being SAW (rule 1) and other SAW-like rules (rules 2, 3 and possibly 4), spiral (rule 7), three-choice (rule 5), two-choice (rule 6(a)) and reverse-two-choice (rule 6(b)). That is, these rules have the most continuing steps, as one might expect-all these rules have, at most four, missing continuing steps. We provide in figure A1 a complete list of all the distinct (up to rotations, reflections and reversals) symmetric-balanced rules starting with the rules with the most continuing steps. In constructing this list we have found a new walk rule, which we informally call the anti-spiral (rule 4). From our enumerations we have placed this model tentatively in class $\mathrm{S}$, although another possibility is that it belongs to a novel universality class. On the other hand, others [30] argue that this rule should be in class D. We are currently analysing longer enumerations of this rule $\dagger[31,32]$. A summary of the universality classes of the contents of the figure $\mathrm{A} 1$ is given in table $\mathrm{A} 1$.

Table A1. The 80 two-dimensional balanced and reverse-balanced walk rules form a total of seven (possibly eight) universality classes. The anti-spiral (rule 4) may be either in a novel universality class $(N)$ or be a slowly converged element of the unrestricted SAW $(S)$ class.

\begin{tabular}{lllcl}
\hline Universality class & $v_{\|}$ & $v_{\perp}$ & Number of rules & Distinct rules \\
\hline SAW $(S)$ & $\frac{3}{4}$ & $\frac{3}{4}$ & 4 & 3 \\
Anti-spiral $(N)$ & $\approx 0.8$ & $\approx 0.6$ & 4 & 1 \\
ASSAW $(A)$ & $0.955(2)$ & $0.4775(10)$ & 12 & 2 \\
Spiral $(P)$ & $\frac{1}{2}(\log )$ & $\frac{1}{2}(\log )$ & 2 & 1 \\
DW $(D)$ & 1 & $\frac{1}{2}$ & 6 & 2 \\
Pseudo-1D $(U)$ & 1 & 1 & 19 & 5 \\
1D $(O)$ & 1 & 0 & 24 & 6 \\
Trivial $(T)$ & 0 & 0 & 9 & 4 \\
\hline
\end{tabular}

$\uparrow$ Note added in proof. Series analysis of the longest series generated (50 terms) seems to indicate that anti-spirals is a separate universality class. However, our preliminary Monte Carlo results up to length 8192 imply that the rule may indeed be directed, or close to it, which in turn implies that a slight modification of the 'mixing' condition may be necessary in two dimensions. 
(S)

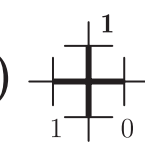
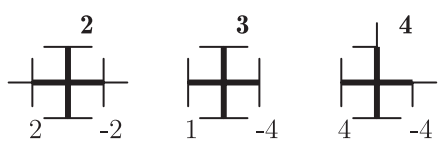

(A)

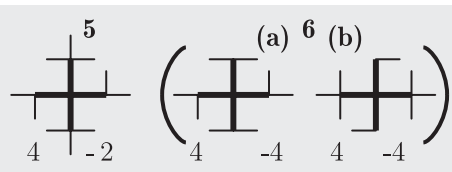

(P) $\frac{t^{7}}{\sqrt{t_{-4}}}$

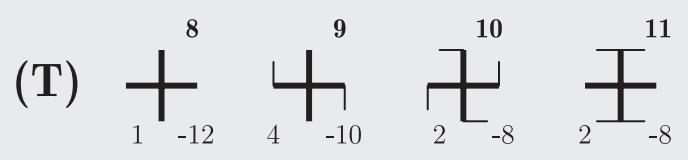

(0)
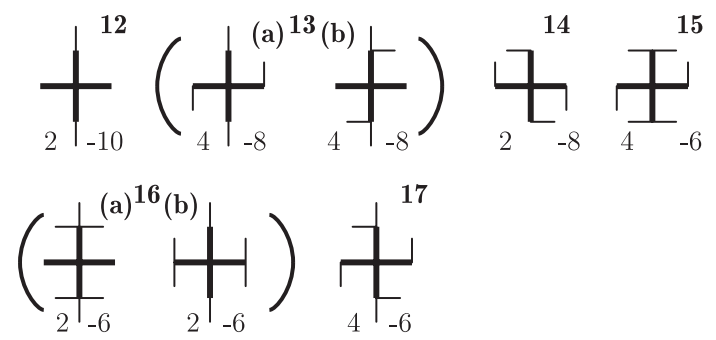

(U)
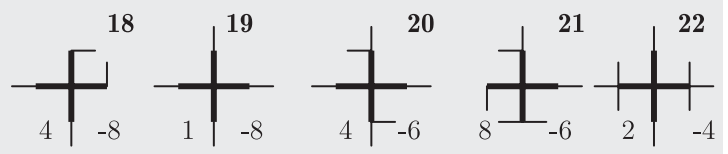

(D)

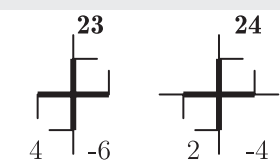

Figure A1. Diagrams of all 24 distinct (up to rotations, reflections and reversals) balanced TSRW rules on the square lattice. The rules are classified according to their apparent universality class. Each pair of rules 6(a) and 6(b), rules 13(a) and 13(b), and rules 16(a) and 16(b) are simply reversals (traversing the rule backwards) of each other respectively. We have assigned each distinct rule a number label (in bold) above each pictogram, and indicated below each rule both the degeneracy of the rule on the left and on the right the number of continuing steps that have to be removed from the unrestricted SAW rule to give that rule (e.g. -4 means four steps need to be removed).

\section{Appendix B. Number of symmetric-balanced rules}

\section{Appendix B.1. Calculating the total number of balanced rules}

For a rule to be balanced we require that there are the same number of continuing steps in the positive component as in the negative component of each axial direction. On the $d$-dimensional hypercubic lattice there are a total of $2 d-1$ different possible continuing steps in each of the positive and negative components of each axial direction. 
To obtain a balanced rule with $k$ continuing steps in some positive axial direction we hence require that there be $k$ continuing steps in the negative axial direction. There are $\left(\begin{array}{c}2 d-1 \\ k\end{array}\right)$ ways of choosing the former set of $k$ steps, and the same number of ways of choosing the latter set of $k$ steps. Hence

$$
\text { the number of choices of } k \text { pairs of steps }=\left(\begin{array}{c}
2 d-1 \\
k
\end{array}\right)^{2} \text {. }
$$

We can sum this over $k$ to get the number of rules that are balanced with respect to that axis:

$$
\sum_{k=0}^{2 d-1}\left(\begin{array}{c}
2 d-1 \\
k
\end{array}\right)^{2}=\left(\begin{array}{c}
4 d-2 \\
2 d-1
\end{array}\right)
$$

Since there are $d$ directions on the hypercubic lattice we obtain the following result:

$$
\text { the number of balanced rules on the hypercubic lattice }=\left(\begin{array}{c}
4 d-2 \\
2 d-1
\end{array}\right)^{d} \text {. }
$$

However, there are rules that are balanced that are not reverse-balanced, and hence we wish to calculate the cardinality of the symmetric-balanced rule space.

Appendix B.2. Calculating the number of symmetric-balanced rules sorted according to the number of TSCs

In this section we do two things simultaneously: we count the number of symmetric-balanced rules and further we sort them according to the number of TSCs the rule contains. To do this we will introduce suitable generating functions. To accomplish the first task we make use of MacMahon's $\stackrel{\lambda}{\Omega}$ operator [33], which is a 'constant term' operator (see below).

We show here in detail the analysis for square lattice TSRW and then just state the result for higher-dimensional hyper-cubic lattices. We first write the $M$ matrix for two dimensions (in the manner described in section 2) as

$$
M=\left[\begin{array}{cccc}
r r & 0 & r f & r b \\
0 & l l & l f & l b \\
f r & f l & f f & 0 \\
b r & b l & 0 & b b
\end{array}\right]
$$

with each variable entry taking the value of 0 or 1 . That is, the TSRW rules are in bijection with matrices with binary entries constrained by the condition that the elements in the positions $(1,2),(2,1),(3,4)$ and $(4,3)$ are fixed to be 0 . Let us call the set of all such matrices $\mathcal{M}$. The symmetric-balance conditions for the square lattice are given by

$$
\sum_{i} M_{i, 2 j-1}=\sum_{i} M_{i, 2 j} \quad j=1,2
$$

and

$$
\sum_{i} M_{2 j-1, i}=\sum_{i} M_{2 j, i} \quad j=1,2 .
$$

That is, we require the sum of the first two rows (columns) to be equal and the sum of the second two rows (columns) to be equal.

To be able to sort rules according to the number of TSCs we introduce a generating function whose variable $t$ counts the number of TSCs. As an example, we can easily write down the TSC generating function $g_{\text {all }}(t)$ for all rules. Let us assign a variable, $t$, to each TSC in the rule 
(which is equivalent to assigning a variable to each non-zero element of $M$ ). The generating function is obtained by summing over all the possible allowed matrices $M$, that is $M \in \mathcal{M}$ :

$$
\begin{aligned}
g_{\text {all }}(t) & =\sum_{M \in \mathcal{M}} \prod_{(i, j)} t^{M_{i, j}} \\
& =\prod_{(\text {allowed } i, j)} \sum_{M_{i, j}=0,1} t^{M_{i, j}}=(1+t)^{(\text {\#allowed } i, j)} \\
& =(1+t)^{12}=\sum_{k=1}^{12}\left(\begin{array}{c}
12 \\
k
\end{array}\right) t^{k}
\end{aligned}
$$

We can impose the symmetric-balance conditions by associating an additional weight with each element of $M$ and then taking the constant term of the resultant polynomials with respect to these variables. To illustrate the method in detail, we first consider the number of balanced rules sorted according to the number of TSCs. This generalizes the result of the previous section. The TSC generating function $g_{\text {bal }}(t)$ we require is then

$$
g_{\text {bal }}(t)=\sum_{M \in \mathcal{M} \mid\left(\sum_{i} M_{i, 1}=\sum_{i}\right.} \prod_{M_{i, 2}} \text { and } \sum_{\sum_{i}} t_{i, 3} \sum^{M_{i, j}} .
$$

We now show that this generating function $g_{\text {bal }}(t)$ can be written as the constant term of a generalized generating function $P\left(\mu_{x}, \mu_{y} ; t\right)$ with respect to the new variables $\mu_{x}$ and $\mu_{y}$. Taking the constant term of such a series is described by the action of the operators $\underset{\mu_{x}}{=}$ and $\underset{\mu_{y}}{\Omega}$ defined by MacMahon [33]. The operator $\stackrel{\lambda}{\Omega}$ acts on a series $f(\lambda)$ so as to remove any term in which $\lambda$ appears: i.e. finds the constant term (with respect to $\lambda$ ) of the expression upon which it acts. For example,

$$
\stackrel{\lambda}{\Omega}\left\{(1+x \lambda)^{2}\left(1+\frac{x}{\lambda}\right)^{2}\right\}=1+4 x^{2}+x^{4} .
$$

In our work all the series we consider are finite: that is, they are polynomials.

We begin by rewriting the balance constraint on the sum over the rules as a Kronecker $\delta$ factor acting on an unconstrained sum. Hence we have

$$
g_{\text {bal }}(t)=\sum_{M \in \mathcal{M}}\left[\prod_{i, j} t^{M_{i, j}} \delta\left(\sum_{j} M_{1, j}-\sum_{j} M_{2, j}\right) \delta\left(\sum_{j} M_{3, j}-\sum_{j} M_{4, j}\right)\right] .
$$

The Kronecker $\delta$ picks out of the unconstrained (finite) sum the terms satisfying the conditions. Another way to evaluate a finite sum of the form $\sum_{j} a_{j} \delta(f(j))$ is to introduce a variable that 'counts' deviations from this constraint and takes the constant term of the resulting function. That is,

$$
\sum_{j} a_{j} \delta(f(j))=\text { constant term of } \lambda \text { in } \sum_{j} a_{j} \lambda^{f(j)} .
$$

Introducing variables $\mu_{x}$ and $\mu_{y}$ to take account of the two constraints we obtain

$$
\begin{aligned}
g_{\text {bal }}(t) & =\stackrel{\mu_{x} \mu_{y}}{\Omega}=\left\{\sum_{M \in \mathcal{M}}\left(\prod_{i, j} t^{M_{i, j}} \mu_{x}^{\sum_{j} M_{1, j}-\sum_{j} M_{2, j}} \mu_{y}^{\sum_{j} M_{3, j}-\sum_{j} M_{4, j}}\right)\right\} \\
& =\stackrel{\mu_{x} \mu_{y}}{\Omega}\left\{P\left(\mu_{x}, \mu_{y} ; t\right)\right\} \\
& =
\end{aligned}
$$

which also provides the definition of the function $P\left(\mu_{x}, \mu_{y} ; t\right)$. Note that $P\left(\mu_{x}, \mu_{y} ; t\right)$ can be constructed by forming a generating function from the set $\mathcal{M}$ by weighting the (non-zero) 
elements in column 1 by $\mu_{x}$ and those in column 2 by $1 / \mu_{x}$ and weighting the (non-zero) elements in column 3 by $\mu_{y}$ and those in column 4 by $1 / \mu_{y}$, as well as weighting any (nonzero) element by $t$. Now we can easily evaluate $P\left(\mu_{x}, \mu_{y} ; t\right)$ as a product in the same way we previously found $g_{\text {all }}(t)$. Hence, examining the operand $P\left(\mu_{x}, \mu_{y} ; t\right)$ in more detail gives

$$
\begin{aligned}
P\left(\mu_{x}, \mu_{y} ; t\right) & =\sum_{M \in \mathcal{M}}\left(\prod_{j}\left(\mu_{x} t\right)^{M_{1, j}}\left(\frac{t}{\mu_{x}}\right)^{M_{2, j}}\left(\mu_{y} t\right)^{M_{3, j}}\left(\frac{t}{\mu_{y}}\right)^{M_{4, j}}\right) \\
& =\left(1+\mu_{x} t\right)^{3}\left(1+\frac{t}{\mu_{x}}\right)^{3}\left(1+\mu_{y} t\right)^{3}\left(1+\frac{t}{\mu_{y}}\right)^{3} .
\end{aligned}
$$

In this case one can explicitly evaluate the constant term as

$$
\begin{aligned}
g_{\text {bal }}(t) & =\underset{=}{\mu_{x}}\left\{\left(\sum_{k=0}^{3}\left(\begin{array}{l}
3 \\
k
\end{array}\right) \mu_{x} t^{k}\right)\left(\sum_{k=0}^{3}\left(\begin{array}{l}
3 \\
k
\end{array}\right) \frac{t^{k}}{\mu_{x}}\right)\right\} \times \underset{=}{\Omega}\left\{\left(\sum_{k=0}^{3}\left(\begin{array}{l}
3 \\
k
\end{array}\right) \mu_{y} t^{k}\right)\left(\sum_{k=0}^{3}\left(\begin{array}{l}
3 \\
k
\end{array}\right){\frac{t^{k}}{\mu_{y}}}^{k}\right)\right\} \\
& =\left(\sum_{k=0}^{3} t^{2 k}\left(\begin{array}{l}
3 \\
k
\end{array}\right)^{2}\right)^{2} .
\end{aligned}
$$

The coefficient of $t^{n}$ is the number of balanced configurations of $M$ with $n$ TSCs. Note that putting $t=1$ recovers the result (B.3) in the case $d=2$.

We now consider the generating function of rules that are symmetric-balanced in two dimensions. We define a generating function that counts such rules according to the number of TSCs as

$$
g_{2, \mathrm{sym}-\mathrm{bal}}(t)=\sum_{M \in \mathcal{M}}^{*} \prod_{i, j} t^{M_{i, j}}
$$

where the $*$ on the sum means that the sum is constrained by both conditions (B.5) and (B.6). Again this can be written as a multiple constant term expression,

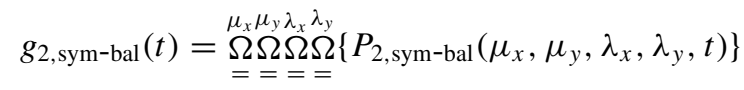

where

$$
\begin{aligned}
P_{2, \text { sym-bal }}\left(\mu_{x},\right. & \left.\mu_{y}, \lambda_{x}, \lambda_{y} ; t\right)=\left(1+t \mu_{x} \lambda_{x}\right)\left(1+t \mu_{y} \lambda_{x}\right)\left(1+\frac{t \lambda_{x}}{\mu_{y}}\right) \\
& \times\left(1+\frac{t}{\mu_{x} \lambda_{x}}\right)\left(1+\frac{t \mu_{y}}{\lambda_{x}}\right)\left(1+\frac{t}{\mu_{y} \lambda_{x}}\right) \\
& \times\left(1+t \mu_{x} \lambda_{y}\right)\left(1+\frac{t \lambda_{y}}{\mu_{x}}\right)\left(1+t \mu_{y} \lambda_{y}\right) \\
& \times\left(1+\frac{t \mu_{x}}{\lambda_{y}}\right)\left(1+\frac{t}{\mu_{x} \lambda_{y}}\right)\left(1+\frac{t}{\mu_{y} \lambda_{y}}\right) .
\end{aligned}
$$

Using Maple $\mathrm{T}^{\mathrm{TM}} \dagger$ to expand the polynomial and take the constant term, we find that

$$
g_{2, \text { sym-bal }}(t)=1+6 t^{2}+19 t^{4}+28 t^{6}+19 t^{8}+6 t^{10}+t^{12}
$$

As constructed, the coefficient of $t^{n}$ represents the number of symmetric-balanced TSRW rules made up of $n$ TSCs. This can be explicitly seen, after some counting, from the catalogue of

$\dagger$ Maple $^{\mathrm{TM}}$ is a registered trademark of Waterloo Maple Software. 
appendix A. By a similar process as that outlined above we obtain the following results for three dimensions:

$$
\begin{aligned}
g_{3, \text { sym-bal }}(t)= & \stackrel{\mu_{x}, \mu_{y}, \mu_{z}, \lambda_{x}, \lambda_{y}, \lambda_{z}}{\Omega}\left\{P_{3, \text { sym-bal }}\left(\mu_{x}, \mu_{y}, \mu_{z}, \lambda_{x}, \lambda_{y}, \lambda_{z}, t\right)\right\} \\
= & t^{30}+15 t^{28}+177 t^{26}+1519 t^{24}+8457 t^{22}+30183 t^{20} \\
& +69829 t^{18}+105867 t^{16}+105867 t^{14}+69829 t^{12} \\
& +30183 t^{10}+8457 t^{8}+1519 t^{6}+177 t^{4}+15 t^{2}+1
\end{aligned}
$$

and four dimensions:

$$
\begin{aligned}
g_{4, \text { sym-bal }}(t)= & \stackrel{\mu_{i}, \lambda_{i} ;\{i=1 \ldots 4\}}{\Omega}\left\{P_{4, \text { sym-bal }}\left(\mu_{i}, \lambda_{i}, t\right)\right\} \\
= & t^{56}+28 t^{54}+738 t^{52}+16268 t^{50} \\
& +274907 t^{48}+\cdots+94001836824 t^{28} \\
& +\cdots+274907 t^{8}+16268 t^{6}+738 t^{4}+28 t^{2}+1 .
\end{aligned}
$$

Substituting $t=1$ into the generating functions gives the total number of symmetric-

\begin{tabular}{|c|c|c|c|c|c|c|}
\hline \multirow[b]{2}{*}{$n$} & \multicolumn{2}{|c|}{$(\operatorname{Rot}-\pi)$} & \multicolumn{2}{|c|}{$(P-P-3)$} & \multicolumn{2}{|c|}{$(P-P-D)$} \\
\hline & $c_{n}$ & $\left\langle R_{g}^{2}\right\rangle_{n}$ & $c_{n}$ & $\left\langle R_{g}^{2}\right\rangle_{n}$ & $c_{n}$ & $\left\langle R_{g}^{2}\right\rangle_{n}$ \\
\hline 1 & 6 & 0.2500000000 & 6 & 0.250000 & 6 & 0.2500000000 \\
\hline 2 & 18 & 0.5185185185 & 20 & 0.511111 & 18 & 0.5185185185 \\
\hline 3 & 54 & 0.7870370370 & 66 & 0.772727 & 54 & 0.7962962963 \\
\hline 4 & 158 & 1.062278481 & 206 & 1.060971 & 154 & 1.112727273 \\
\hline 5 & 466 & 1.330591321 & 652 & 1.352079 & 446 & 1.444319880 \\
\hline 6 & 1338 & 1.616180104 & 2012 & 1.667018 & 1266 & 1.810233098 \\
\hline 7 & 3886 & 1.894042717 & 6264 & 1.984924 & 3626 & 2.189878654 \\
\hline 8 & 11082 & 2.189255850 & 19254 & 2.321767 & 10282 & 2.598303160 \\
\hline 9 & 31842 & 2.479427800 & 59442 & 2.662321 & 29262 & 3.020260406 \\
\hline 10 & 90542 & 2.782749105 & 182148 & 3.018780 & 82790 & 3.467095379 \\
\hline 11 & 258466 & 3.083967846 & 559568 & 3.378845 & 234674 & 3.925950941 \\
\hline 12 & 733190 & 3.395616965 & 1710476 & 3.752893 & 662518 & 4.406914942 \\
\hline 13 & 2084726 & 3.706346879 & 5237592 & 4.130143 & 1872906 & 4.897985755 \\
\hline 14 & 5902350 & 4.026035244 & 15980914 & 4.519812 & 5279098 & 5.408667159 \\
\hline 15 & 16738270 & 4.345208279 & 48822216 & 4.912359 & 14895186 & 5.927984092 \\
\hline 16 & 47319166 & 4.672362374 & 148757054 & 5.316068 & 41934586 & 6.464774052 \\
\hline 17 & 133935010 & 4.999214463 & 453683704 & 5.722368 & 118150286 & 7.009094165 \\
\hline 18 & 378189902 & 5.333289679 & 1380795336 & 6.138830 & 332311930 & 7.569169389 \\
\hline 19 & 1068895606 & 5.667227224 & 4205655986 & 6.557627 & 935234466 & 8.135866302 \\
\hline 20 & 3015366794 & 6.007756322 & 12788431474 & 6.985764 & 2628389278 & 8.716905437 \\
\hline
\end{tabular}
balanced rules. Hence find that there are

- 80 TSRW rules in two dimensions,

- 432096 TSRW rules in three dimensions and

- 478340593664 TSRW rules in four dimensions.

\section{Appendix C. Exact enumeration data}

Appendix C.1. Radius of gyration and number of walk configuration tables

Table C1. The enumeration data, $c_{n}$ and $\left\langle R_{g}^{2}\right\rangle$, for the rules (Rot- $\left.\pi\right),(P-P-3)$ and $(P-P-D)$. 
Table C1. (Continued)

\begin{tabular}{|c|c|c|c|c|c|c|}
\hline \multirow[b]{2}{*}{$n$} & \multicolumn{2}{|c|}{$(\operatorname{Rot}-\pi)$} & \multicolumn{2}{|c|}{$(P-P-3)$} & \multicolumn{2}{|c|}{$(P-P-D)$} \\
\hline & $c_{n}$ & $\left\langle R_{g}^{2}\right\rangle_{n}$ & $c_{n}$ & $\left\langle R_{g}^{2}\right\rangle_{n}$ & $c_{n}$ & $\left\langle R_{g}^{2}\right\rangle_{n}$ \\
\hline 21 & 8512718274 & 6.348276986 & 38910558974 & 7.416014 & 7390474750 & 9.303800493 \\
\hline 22 & 23995774102 & 6.694862106 & 118229769376 & 7.854911 & 20756622062 & 9.903846043 \\
\hline 23 & & & 359424248858 & 8.295731 & & \\
\hline 24 & & & 1091427480250 & 8.744604 & & \\
\hline 25 & & & 3315651798324 & 9.195238 & & \\
\hline
\end{tabular}

Table C2. The enumeration data, $c_{n}$ and $\left\langle R_{g}^{2}\right\rangle$, for the rules $(S-C-3),(S-C-P),(3-3-C)$ and $(S-P-3)$.

\begin{tabular}{|c|c|c|c|c|c|c|}
\hline \multirow[b]{2}{*}{$n$} & \multicolumn{2}{|c|}{$S-C-3$} & \multicolumn{2}{|c|}{$(S-C-P)$} & \multicolumn{2}{|c|}{$(3-3-C)$} \\
\hline & $c_{n}$ & $\left\langle R_{g}^{2}\right\rangle_{n}$ & $c_{n}$ & $\left\langle R_{g}^{2}\right\rangle_{n}$ & $c_{n}$ & $\left\langle R_{g}^{2}\right\rangle_{n}$ \\
\hline 1 & 6 & 0.250000 & 6 & 0.2500000000 & 6 & 0.250000 \\
\hline 2 & 24 & 0.500000 & 22 & 0.5050505050 & 22 & 0.505051 \\
\hline 3 & 90 & 0.766667 & 78 & 0.7692307690 & 80 & 0.771875 \\
\hline 4 & 324 & 1.059259 & 266 & 1.056842106 & 284 & 1.060845 \\
\hline 5 & 1166 & 1.366447 & 910 & 1.354273505 & 1014 & 1.359358 \\
\hline 6 & 4138 & 1.694716 & 3054 & 1.672266549 & 3564 & 1.678142 \\
\hline 7 & 14730 & 2.031848 & 10310 & 1.993913676 & 12588 & 2.002155 \\
\hline 8 & 51992 & 2.386510 & 34446 & 2.331570689 & 44098 & 2.341145 \\
\hline 9 & 183898 & 2.747547 & 115450 & 2.672526808 & 154832 & 2.684732 \\
\hline 10 & 646980 & 3.122930 & 384530 & 3.026504010 & 540770 & 3.040744 \\
\hline 11 & 2279702 & 3.502933 & 1283462 & 3.382982338 & 1891584 & 3.400228 \\
\hline 12 & 8002976 & 3.895122 & 4265822 & 3.750604402 & 6592486 & 3.770323 \\
\hline 13 & 28127418 & 4.290869 & 14199618 & 4.120239122 & 23001542 & 4.143167 \\
\hline 14 & 98585096 & 4.697309 & 47120838 & 4.499743779 & 80037948 & 4.525304 \\
\hline 15 & 345848306 & 5.106591 & 156545474 & 4.880926243 & 278740232 & 4.909733 \\
\hline 16 & 1210704274 & 5.525411 & 518858122 & 5.270979045 & 968743336 & 5.302460 \\
\hline 17 & 4241348770 & 5.946576 & 1721232166 & 5.662463560 & 3369017390 & 5.697144 \\
\hline 18 & 14833284544 & 6.376375 & 5699369614 & 6.062020580 & 11697449542 & 6.099341 \\
\hline 19 & 51907058582 & 6.808156 & 18885164058 & 6.462824345 & 40635868918 & 6.503236 \\
\hline 20 & 181392476966 & 7.247841 & 62483445082 & 6.871045435 & 140979332596 & 6.914013 \\
\hline 21 & 634197818374 & 7.689232 & 206852302966 & 7.280367595 & & \\
\hline 22 & 2214804822718 & 8.137924 & 683942288222 & 7.696558450 & & \\
\hline 23 & 7737946227490 & 8.588107 & & & & \\
\hline \multicolumn{3}{|c|}{$(S-P-3)$} & & \multicolumn{3}{|c|}{$(S-P-3)$ (continued) } \\
\hline$n$ & $c_{n}$ & $\left\langle R_{g}^{2}\right\rangle_{n}$ & & $n$ & $c_{n}$ & $\left\langle R_{g}^{2}\right\rangle_{n}$ \\
\hline 1 & 6 & 0.250000 & & 10 & 1105052 & 2.894842 \\
\hline 2 & 24 & 0.500000 & & 11 & 4165768 & 3.232301 \\
\hline 3 & 96 & 0.752604 & & 12 & 15635564 & 3.580525 \\
\hline 4 & 368 & 1.029130 & & 13 & 58773288 & 3.930671 \\
\hline 5 & 1422 & 1.311260 & & 14 & 220229536 & 4.290298 \\
\hline 6 & 5392 & 1.612548 & & 15 & 826135272 & 4.651580 \\
\hline 7 & 20562 & 1.917156 & & 16 & 3091645402 & 5.021351 \\
\hline 8 & 77590 & 2.237052 & & 17 & 11579713514 & 5.392576 \\
\hline 9 & 293760 & 2.559677 & & 18 & 43290642466 & 5.771503 \\
\hline
\end{tabular}


Table C3. The enumeration data, $c_{n}$ and $\left\langle R_{g}^{2}\right\rangle$, for the rules $(P-O-3),(P-3-3)$ and $(P-2-2)$.

\begin{tabular}{|c|c|c|c|c|c|c|}
\hline \multirow[b]{2}{*}{$n$} & \multicolumn{2}{|c|}{$(P-O-3)$} & \multicolumn{2}{|c|}{$(P-3-3)$} & \multicolumn{2}{|c|}{$(P-2-2)$} \\
\hline & $c_{n}$ & $\left\langle R_{g}^{2}\right\rangle_{n}$ & $c_{n}$ & $\left\langle R_{g}^{2}\right\rangle_{n}$ & $c_{n}$ & $\left\langle R_{g}^{2}\right\rangle_{n}$ \\
\hline 1 & 6 & 0.2500000000 & 6 & 0.250000 & 3 & 0.250000 \\
\hline 2 & 16 & 0.5277777780 & 22 & 0.505051 & 10 & 0.488889 \\
\hline 3 & 44 & 0.8125000000 & 78 & 0.769231 & 32 & 0.734375 \\
\hline 4 & 112 & 1.147142857 & 266 & 1.056842 & 98 & 1.002449 \\
\hline 5 & 290 & 1.489080460 & 914 & 1.351872 & 302 & 1.275754 \\
\hline 6 & 730 & 1.862230920 & 3072 & 1.670095 & 905 & 1.572804 \\
\hline 7 & 1858 & 2.238125673 & 10388 & 1.992612 & 2731 & 1.872414 \\
\hline 8 & 4644 & 2.641571230 & 34696 & 2.333659 & 8121 & 2.190149 \\
\hline 9 & 11692 & 3.045703045 & 116326 & 2.677870 & 24254 & 2.510089 \\
\hline 10 & 29128 & 3.471307038 & 387094 & 3.037176 & 71801 & 2.844642 \\
\hline 11 & 72866 & 3.898065933 & 1291232 & 3.399126 & 213168 & 3.181094 \\
\hline 12 & 181092 & 4.342164570 & 4285502 & 3.773997 & 629044 & 3.530483 \\
\hline 13 & 451246 & 4.787541630 & 14247504 & 4.151048 & 1860191 & 3.881363 \\
\hline 14 & 1119492 & 5.247478820 & 47195288 & 4.539472 & 5476975 & 4.243746 \\
\hline 15 & 2782326 & 5.708806865 & 156532896 & 4.929770 & 16151357 & 4.607443 \\
\hline 16 & 6893472 & 6.182730795 & 517758628 & 5.330238 & 47474808 & 4.981465 \\
\hline 17 & 17101294 & 6.658119150 & 1714230392 & 5.732355 & 139716218 & 5.356659 \\
\hline 18 & 42325616 & 7.144718770 & 5663536142 & 6.143698 & 410143001 & 5.741247 \\
\hline 19 & 104857966 & 7.632773830 & 18725774596 & 6.556509 & 1205156331 & 6.126905 \\
\hline 20 & 259302146 & 8.131019980 & 61809095034 & 6.977778 & 3534113551 & 6.521186 \\
\hline 21 & 641714298 & 8.630686255 & 204143781018 & 7.400373 & 10371867145 & 6.916460 \\
\hline 22 & 1585767184 & 9.139738495 & 673309667636 & 7.830787 & 30389668612 & 7.319704 \\
\hline 23 & 3921055588 & 9.650150405 & 2221867592366 & 8.262410 & 89099312437 & 7.723878 \\
\hline 24 & 9683676170 & 10.16930800 & 7323479170494 & 8.701308 & 260877974233 & 8.135467 \\
\hline 25 & 23927486656 & 10.68974044 & & & & \\
\hline 26 & 59062356252 & 11.21838694 & & & & \\
\hline 27 & 145850988968 & 11.74822225 & & & & \\
\hline 28 & 359856328600 & 12.28581177 & & & & \\
\hline
\end{tabular}

Table C4. The enumeration data, $c_{n}$ and $\left\langle R_{g}^{2}\right\rangle$, for the rules $(P-P-3),(P-R-2)$ and $(P-P-P)$.

\begin{tabular}{|c|c|c|c|c|c|c|}
\hline \multirow[b]{2}{*}{$n$} & \multicolumn{2}{|c|}{$(P-P-3)$} & \multicolumn{2}{|c|}{$(P-R-2)$} & \multicolumn{2}{|c|}{$(P-P-P)$} \\
\hline & $c_{n}$ & $\left\langle R_{g}^{2}\right\rangle_{n}$ & $c_{n}$ & $\left\langle R_{g}^{2}\right\rangle_{n}$ & $c_{n}$ & $\left\langle R_{g}^{2}\right\rangle_{n}$ \\
\hline 1 & 6 & 0.250000 & 6 & 0.5000000000 & 6 & 0.250000 \\
\hline 2 & 20 & 0.511111 & 18 & 0.9876543210 & 18 & 0.518519 \\
\hline 3 & 66 & 0.772727 & 54 & 1.472222222 & 54 & 0.777778 \\
\hline 4 & 206 & 1.060971 & 150 & 2.018133333 & 150 & 1.059200 \\
\hline 5 & 652 & 1.352079 & 426 & 2.565206051 & 426 & 1.325117 \\
\hline 6 & 2012 & 1.667018 & 1170 & 3.165532880 & 1158 & 1.613620 \\
\hline 7 & 6264 & 1.984924 & 3250 & 3.769846154 & 3204 & 1.891912 \\
\hline 8 & 19254 & 2.321767 & 8890 & 4.413814940 & 8682 & 2.187736 \\
\hline 9 & 59442 & 2.662321 & 24444 & 5.064869907 & 23724 & 2.481578 \\
\hline 10 & 182148 & 3.018780 & 66598 & 5.750268231 & 64194 & 2.788945 \\
\hline
\end{tabular}


Table C4. (continued)

\begin{tabular}{|c|c|c|c|c|c|c|}
\hline \multirow[b]{2}{*}{$n$} & \multicolumn{2}{|c|}{$(P-P-3)$} & \multicolumn{2}{|c|}{$(P-R-2)$} & \multicolumn{2}{|c|}{$(P-P-P)$} \\
\hline & $c_{n}$ & $\left\langle R_{g}^{2}\right\rangle_{n}$ & $c_{n}$ & $\left\langle R_{g}^{2}\right\rangle_{n}$ & $c_{n}$ & $\left\langle R_{g}^{2}\right\rangle_{n}$ \\
\hline 11 & 559568 & 3.378845 & 182044 & 6.442183392 & 174378 & 3.097380 \\
\hline 12 & 1710476 & 3.752893 & 494462 & 7.165571886 & 470856 & 3.418129 \\
\hline 13 & 5237592 & 4.130143 & 1346212 & 7.894713402 & 1274430 & 3.740403 \\
\hline 14 & 15980914 & 4.519812 & 3648594 & 8.652187898 & 3434826 & 4.074125 \\
\hline 15 & 48822216 & 4.912359 & 9905610 & 9.415095218 & 9272346 & 4.409709 \\
\hline 16 & 148757054 & 5.316068 & 26803048 & 10.20352410 & 24953004 & 4.755764 \\
\hline 17 & 453683704 & 5.722368 & 72618674 & 10.99703701 & 67230288 & 5.103799 \\
\hline 18 & 1380795336 & 6.138830 & 196243194 & 11.81383270 & 180705126 & 5.461477 \\
\hline 19 & 4205655986 & 6.557627 & 530861042 & 12.63534041 & 486152604 & 5.821086 \\
\hline 20 & 12788431474 & 6.985764 & 1433106984 & 13.47830840 & 1305430884 & 6.189624 \\
\hline 21 & 38910558974 & 7.416014 & 3871966934 & 14.32562644 & 3507947838 & 6.560016 \\
\hline 22 & 118229769376 & 7.854911 & 10443886572 & 15.19285413 & 9412114986 & 6.938709 \\
\hline 23 & 359424248858 & 8.295731 & 28189364062 & 16.06411132 & 25268587338 & 7.319174 \\
\hline 24 & 1091427480250 & 8.744604 & 75981934450 & 16.95392965 & 67752451146 & 7.707392 \\
\hline 25 & 3315651798324 & 9.195238 & 204918079282 & 17.84749654 & 181754458194 & 8.097294 \\
\hline 26 & & & 552010606124 & 18.75844867 & 487060621596 & 8.494472 \\
\hline 27 & & & & & 1305761069730 & 8.893243 \\
\hline 28 & & & & & 3497441209182 & 9.298868 \\
\hline 29 & & & & & 9371171057352 & 9.705999 \\
\hline
\end{tabular}

Appendix C.2. Moment of inertia eigenvalue enumeration tables

Table C5. Eigenvalues of the moment of inertia tensor computed about the centre of mass for the $(S-C-P)$ and $(P-O-3)$ rules.

\begin{tabular}{|c|c|c|c|c|c|c|}
\hline \multirow[b]{2}{*}{$n$} & \multicolumn{3}{|c|}{$(S-C-P)$} & \multicolumn{3}{|c|}{$(P-O-3)$} \\
\hline & $\lambda_{1}$ & $\lambda_{2}$ & $\lambda_{3}$ & $\lambda_{1}$ & $\lambda_{2}$ & $\lambda_{3}$ \\
\hline 1 & 0.1666666667 & 0.1666666667 & 0.1666666667 & 0.166666666667 & 0.166666666667 & 0.166666666667 \\
\hline 2 & 0.3232323232 & 0.3232323232 & 0.3636363636 & 0.302276833646 & 0.364389833021 & 0.388888888889 \\
\hline 3 & 0.4839743590 & 0.5836984453 & 0.4707887341 & 0.426824397156 & 0.569876200940 & 0.628299401917 \\
\hline 4 & 0.6556390977 & 0.8281265757 & 0.6299185371 & 0.566948065248 & 0.814084994660 & 0.913252654398 \\
\hline 5 & 0.8321123321 & 1.083682865 & 0.7927518113 & 0.698817526155 & 1.07055653765 & 1.20878685577 \\
\hline 6 & 1.018670730 & 1.358246075 & 0.9676162926 & 0.834240876666 & 1.35502001927 & 1.53520094359 \\
\hline 7 & 1.206237876 & 1.636945906 & 1.144643570 & 0.965014568266 & 1.64539790396 & 1.86583887337 \\
\hline 8 & 1.401436351 & 1.930158848 & 1.331546178 & 1.09943234575 & 1.95967965142 & 2.22403046199 \\
\hline 9 & 1.597762148 & 2.226718876 & 1.520572592 & 1.23112509025 & 2.27586578203 & 2.58441521738 \\
\hline 10 & 1.800378395 & 2.535047728 & 1.717581896 & 1.36639773324 & 2.60995149821 & 2.96626484468 \\
\hline 11 & 2.003823794 & 2.845912484 & 1.916228398 & 1.50016239182 & 2.94527512399 & 3.35069434886 \\
\hline 12 & 2.212733910 & 3.166808488 & 2.121666404 & 1.63751026726 & 3.29451156412 & 3.75230730827 \\
\hline 13 & 2.422322095 & 3.489717948 & 2.328438200 & 1.77386437145 & 3.64475300486 & 4.15646588292 \\
\hline 14 & 2.636818108 & 3.821491853 & 2.541177597 & 1.91361659394 & 4.00636209865 & 4.57497894901 \\
\hline 15 & 2.851890731 & 4.154931390 & 2.755030364 & 2.05268553596 & 4.36899823418 & 4.99592995707 \\
\hline
\end{tabular}


Table C5. (Continued)

\begin{tabular}{|c|c|c|c|c|c|c|}
\hline \multirow[b]{2}{*}{$n$} & \multicolumn{3}{|c|}{$(S-C-P)$} & \multicolumn{3}{|c|}{$(P-O-3)$} \\
\hline & $\lambda_{1}$ & $\lambda_{2}$ & $\lambda_{3}$ & $\lambda_{1}$ & $\lambda_{2}$ & $\lambda_{3}$ \\
\hline 16 & 3.071423508 & 4.496325628 & 2.974208952 & 2.19487704734 & 4.74131512204 & 5.42926942078 \\
\hline 17 & 3.291456083 & 4.839133151 & 3.194337891 & 2.33659198558 & 5.11473588958 & 5.86491042151 \\
\hline 18 & 3.515583085 & 5.189171582 & 3.419286494 & 2.48117103722 & 5.49671470589 & 6.31155179334 \\
\hline 19 & 3.740151811 & 5.540434799 & 3.645062085 & 2.62540633802 & 5.87982499605 & 6.76031632378 \\
\hline 20 & 3.968508782 & 5.898336079 & 3.875246007 & 2.77228050171 & 6.27070584816 & 7.21905361238 \\
\hline 21 & 4.197260774 & 6.257314900 & 4.106159520 & 2.91890872388 & 6.66271834526 & 7.67974543712 \\
\hline 22 & 4.429540412 & 6.622436345 & 4.341140145 & 3.06797945586 & 7.06190067532 & 8.14959685536 \\
\hline 23 & & & & 3.21687407350 & 7.46218518484 & 8.62124155310 \\
\hline 24 & & & & 3.36804906946 & 7.86917066793 & 9.10139626525 \\
\hline 25 & & & & 3.51909521920 & 8.27720172517 & 9.58318392935 \\
\hline 26 & & & & 3.67228451329 & 8.69154694518 & 10.0729424218 \\
\hline 27 & & & & 3.82538054292 & 9.10687438372 & 10.5641895689 \\
\hline 28 & & & & 3.98050021705 & 9.52818050950 & 11.0629428101 \\
\hline
\end{tabular}

Table C6. Eigenvalues of the moment of inertia tensor computed about the centre of mass for the $(P-2-2)$ and $(P-R-2)$ rules.

\begin{tabular}{|c|c|c|c|c|c|c|}
\hline \multirow[b]{2}{*}{$n$} & \multicolumn{3}{|c|}{$(P-2-2)$} & \multicolumn{3}{|c|}{$(P-R-2)$} \\
\hline & $\lambda_{1}$ & $\lambda_{2}$ & $\lambda_{3}$ & $\lambda_{1}$ & $\lambda_{2}$ & $\lambda_{3}$ \\
\hline 1 & 0.1666666667 & 0.1666666667 & 0.1666666667 & 0.1666666667 & 0.1666666667 & 0.1666666667 \\
\hline 2 & 0.3061167806 & 0.3111111111 & 0.3605498861 & 0.2942928684 & 0.3209876543 & 0.3723737985 \\
\hline 3 & 0.4380603326 & 0.4570312500 & 0.5736584178 & 0.4116361451 & 0.4769444645 & 0.5836416133 \\
\hline 4 & 0.5768549300 & 0.6171428575 & 0.8109001732 & 0.5335997647 & 0.6539704927 & 0.8305630769 \\
\hline 5 & 0.7147827913 & 0.7801507133 & 1.056574958 & 0.6515748546 & 0.8336572627 & 1.079973934 \\
\hline 6 & 0.8619902703 & 0.9586341077 & 1.324983923 & 0.7745233588 & 1.034225881 & 1.356783641 \\
\hline 7 & 1.008795944 & 1.138534983 & 1.597496983 & 0.8959786064 & 1.237000753 & 1.636866796 \\
\hline 8 & 1.162874755 & 1.330319099 & 1.887103536 & 1.021468006 & 1.455531410 & 1.936815524 \\
\hline 9 & 1.317037920 & 1.523442032 & 2.179698169 & 1.146692636 & 1.677217902 & 2.240959373 \\
\hline 10 & 1.477216020 & 1.725865771 & 2.486202909 & 1.276102492 & 1.912047787 & 2.562117957 \\
\hline 11 & 1.637644958 & 1.929534679 & 2.795008619 & 1.405388728 & 2.149897142 & 2.886897521 \\
\hline 12 & 1.803541690 & 2.141291486 & 3.116133542 & 1.538733190 & 2.399671785 & 3.227166913 \\
\hline 13 & 1.969670042 & 2.354060265 & 3.438996579 & 1.672079918 & 2.652093350 & 3.570540133 \\
\hline 14 & 2.140712992 & 2.573986173 & 3.772793127 & 1.809124263 & 2.915259538 & 3.927804101 \\
\hline 15 & 2.312008455 & 2.794817983 & 4.108059365 & 1.946291614 & 3.180868289 & 4.287935315 \\
\hline 16 & 2.487741851 & 3.022057624 & 4.453129695 & 2.086834798 & 3.456143376 & 4.660545924 \\
\hline 17 & 2.663728951 & 3.250112163 & 4.799476692 & 2.227556192 & 3.733680281 & 5.035800539 \\
\hline 18 & 2.843771098 & 3.483990690 & 5.154731719 & 2.371400424 & 4.020017670 & 5.422414610 \\
\hline 19 & 3.024065642 & 3.718614760 & 5.511129042 & 2.515443402 & 4.308440799 & 5.811456215 \\
\hline 20 & 3.208093348 & 3.958584700 & 5.875693123 & 2.662393290 & 4.604966665 & 6.210948447 \\
\hline 21 & 3.392371559 & 4.199246804 & 6.241301327 & 2.809548671 & 4.903410105 & 6.612667668 \\
\hline 22 & 3.580107306 & 4.444850763 & 6.614449240 & 2.959418683 & 5.209367525 & 7.024067925 \\
\hline 23 & 3.768090188 & 4.691102500 & 6.988563927 & 3.109495958 & 5.517093898 & 7.437521480 \\
\hline 24 & 3.959295468 & 4.941953028 & 7.369686415 & 3.262117597 & 5.831822352 & 7.859989694 \\
\hline 25 & & & & 3.414944831 & 6.148190665 & 8.284361061 \\
\hline 26 & & & & 3.570166948 & 6.471113948 & 8.717167785 \\
\hline
\end{tabular}


Table C7. Eigenvalues of the moment of inertia tensor computed about the centre of mass for the $(P-P-P)$ rule. Note that the largest eigenvalue of the $(P-P-P)$ model has degeneracy of 2 .

\begin{tabular}{rll}
\hline & \multicolumn{2}{c}{$(P-P-P)$} \\
\cline { 2 - 3 } & $\lambda_{1}$ & $\lambda_{2}=\lambda_{3}$ \\
\hline 1 & 0.1666666667 & 0.1666666667 \\
2 & 0.3456790123 & 0.3456790123 \\
3 & 0.5092592595 & 0.5231481481 \\
4 & 0.6741333341 & 0.7221333334 \\
5 & 0.8182055301 & 0.9160146058 \\
6 & 0.9626731535 & 1.132282965 \\
7 & 1.094159643 & 1.344832631 \\
8 & 1.225643519 & 1.574914466 \\
9 & 1.352100826 & 1.805527734 \\
10 & 1.479217568 & 2.049336503 \\
11 & 1.604029273 & 2.295365288 \\
12 & 1.730382624 & 2.552937955 \\
13 & 1.855199371 & 2.812803563 \\
14 & 1.981875267 & 3.083187469 \\
15 & 2.107554467 & 3.355931295 \\
16 & 2.235127971 & 3.638200261 \\
17 & 2.362027099 & 3.922785165 \\
18 & 2.490795277 & 4.216079012 \\
19 & 2.619076033 & 4.511547856 \\
20 & 2.749167507 & 4.815040036 \\
21 & 2.878906185 & 5.120562709 \\
22 & 3.010385583 & 5.433515799 \\
23 & 3.141610116 & 5.748368769 \\
24 & 3.274505289 & 6.070139649 \\
25 & 3.407213837 & 6.393687511 \\
26 & 3.541525172 & 6.723709674 \\
27 & 3.675698752 & 7.055393692 \\
28 & 3.811409808 & 7.393163447 \\
\hline & &
\end{tabular}

\section{Appendix D. Tables of fits to scaling forms}

In this section we include the tables of coefficients from the exact local fittings of various scaling forms for both the numbers of walk configurations and the eigenvalues of the moment of inertia tensor.

\section{Appendix D.1. Exact fitting analysis of the number of walk configurations}

In table D1 there are the results of three fits to the numbers of walks, $c_{n}$ for the $(P-P-P)$ model. The top third of the table gives the coefficients of a linear fit using $n, n-2$ and $n-4$ for $n=20, \ldots, 28$ to the form (3.20), which is the canonical scaling form for SAWs. The middle of the table and the bottom give similar fits to the forms (3.21) and (3.22) respectively, using one more term, $n-6$, per fit. In each case the value of the exponent $\gamma$ is allowed to vary. In table D2 the same three fitting forms are used but this time the value of the exponent $\gamma$ is fixed to be the (exact enumeration) SAW estimate of 1.161 at each $n$ (and hence one less term of the series is needed for the fit at each length). In both cases the addition of $\frac{1}{\sqrt{n}}$ or $\frac{1}{n^{2 / 3}}$ corrections only worsen the stability of the other coefficients (slightly). More to the point, the 
coefficients of these corrections are small in all cases and though quite unstable, they appear to be decreasing in magnitude as $n$ increases (but not monotonically). Given their apparent size, it is likely that these corrections are spurious. Whether a value of $\gamma$ closer to 1.16 or 1.19 produces a better fit is a matter for debate. We have included both fits for completeness.

Table D1. Linear fit of the enumerations, $c_{n}$, for the rule $(P-P-P)$, to various scaling forms, with and without corrections.

\begin{tabular}{|c|c|c|c|c|c|}
\hline$n$ & $a_{1}$ & $\log \mu$ & $\gamma-1$ & Corrections & $a_{2}$ \\
\hline 20 & 0.8324756081 & 0.9783192165 & 0.1972603078 & & \\
\hline 21 & 0.8455488938 & 0.9785440966 & 0.1915972483 & & \\
\hline 22 & 0.8373399541 & 0.9784511613 & 0.1947556593 & & \\
\hline 23 & 0.8483239152 & 0.9786137500 & 0.1902053258 & & \\
\hline 24 & 0.8409529395 & 0.9785354534 & 0.1929868688 & & \\
\hline 25 & 0.8503887379 & 0.9786587025 & 0.1892170502 & & \\
\hline 26 & 0.8439695129 & 0.9785969601 & 0.1915731899 & & \\
\hline 27 & 0.8520667855 & 0.9786908435 & 0.1884461032 & & \\
\hline 28 & 0.8466883266 & 0.9786460255 & 0.1903471629 & & \\
\hline 20 & 0.8386930908 & 0.9813251606 & 0.2511716395 & $\sqrt{n}$ & -0.0509466361 \\
\hline 21 & 0.8470176349 & 0.9798194475 & 0.2157468764 & $\sqrt{n}$ & -0.0222091673 \\
\hline 22 & 0.8377676328 & 0.9808777278 & 0.2431470492 & $\sqrt{n}$ & -0.0433633175 \\
\hline 23 & 0.8471827158 & 0.9799644881 & 0.2184954545 & $\sqrt{n}$ & -0.0247359243 \\
\hline 24 & 0.8376594009 & 0.9802556927 & 0.2307406980 & $\sqrt{n}$ & -0.0322466960 \\
\hline 25 & 0.8474684875 & 0.9796235445 & 0.2113569120 & $\sqrt{n}$ & -0.0184932533 \\
\hline 26 & 0.8381955848 & 0.9799756433 & 0.2245944906 & $\sqrt{n}$ & -0.0269970353 \\
\hline 27 & 0.8480120233 & 0.9794441350 & 0.2072396293 & $\sqrt{n}$ & -0.0150543115 \\
\hline 28 & 0.8387374396 & 0.9798462623 & 0.2214956491 & $\sqrt{n}$ & -0.0244635323 \\
\hline 20 & 0.8167669269 & 0.9828759076 & 0.2381152320 & $n^{\frac{2}{3}}$ & -0.0268477019 \\
\hline 21 & 0.8375366772 & 0.9804710583 & 0.2098411804 & $n^{\frac{2}{3}}$ & -0.0115609483 \\
\hline 22 & 0.8193514045 & 0.9821262157 & 0.2313935557 & $n^{\frac{2}{3}}$ & -0.0224305704 \\
\hline 23 & 0.8367633784 & 0.9806570383 & 0.2116019897 & $n^{\frac{2}{3}}$ & -0.0126767502 \\
\hline 24 & 0.8241918436 & 0.9811373146 & 0.2215348590 & $n^{\frac{2}{3}}$ & -0.0163948459 \\
\hline 25 & 0.8398157465 & 0.9801174498 & 0.2059519375 & $n^{\frac{2}{3}}$ & -0.0093291643 \\
\hline 26 & 0.8271044829 & 0.9806847143 & 0.2165707673 & $n^{\frac{2}{3}}$ & -0.0135432894 \\
\hline 27 & 0.8419078052 & 0.9798288918 & 0.2026412139 & $n^{\frac{2}{3}}$ & -0.0074836755 \\
\hline 28 & 0.8289702197 & 0.9804538038 & 0.2138057326 & $n^{\frac{2}{3}}$ & -0.0120453271 \\
\hline
\end{tabular}

Table D2. Linear fit of the enumerations, $c_{n}$, for the rule $(P-P-P)$, to various scaling forms, with and without corrections, and with $\gamma$ fixed at the SAW value.

\begin{tabular}{|c|c|c|c|c|c|}
\hline$n$ & $a_{1}$ & $\log \mu$ & $\gamma-1$ & Corrections & $a_{2}$ \\
\hline 20 & 0.9028978209 & 0.9802294140 & 0.161 & & \\
\hline 21 & 0.9065489941 & 0.9800752350 & 0.161 & & \\
\hline 22 & 0.9062902192 & 0.9800597940 & 0.161 & & \\
\hline 23 & 0.9093432958 & 0.9799421730 & 0.161 & & \\
\hline 24 & 0.9092103892 & 0.9799270590 & 0.161 & & \\
\hline 25 & 0.9118060176 & 0.9798350981 & 0.161 & & \\
\hline 26 & 0.9117668293 & 0.9798205410 & 0.161 & & \\
\hline 27 & 0.9140085907 & 0.9797469950 & 0.161 & & \\
\hline
\end{tabular}


Table D2. (Continued)

\begin{tabular}{llllll}
\hline$n$ & $a_{1}$ & $\log \mu$ & $\gamma-1$ & Corrections & $a_{2}$ \\
\hline 28 & 0.9140311693 & 0.9797334510 & 0.161 & & \\
20 & 0.8282929603 & 0.9762973926 & 0.161 & $\sqrt{n}$ & 0.0342666881 \\
21 & 0.8436960392 & 0.9769286659 & 0.161 & $\sqrt{n}$ & 0.0281350308 \\
22 & 0.8370434575 & 0.9767585843 & 0.161 & $\sqrt{n}$ & 0.0302475055 \\
23 & 0.8494932420 & 0.9772188950 & 0.161 & $\sqrt{n}$ & 0.0255399818 \\
24 & 0.8437391859 & 0.9770777906 & 0.161 & $\sqrt{n}$ & 0.0273227611 \\
25 & 0.8541199161 & 0.9774294158 & 0.161 & $\sqrt{n}$ & 0.0235656305 \\
26 & 0.8493225760 & 0.9773207765 & 0.161 & $\sqrt{n}$ & 0.0249926735 \\
27 & 0.8579899424 & 0.9775908142 & 0.161 & $\sqrt{n}$ & 0.0219846353 \\
28 & 0.8541789041 & 0.9775151871 & 0.161 & $\sqrt{n}$ & 0.0230489648 \\
20 & 0.8464170165 & 0.9742748997 & 0.161 & $n^{\frac{2}{3}}$ & 0.0238286629 \\
21 & 0.8589863846 & 0.9753123551 & 0.161 & $n^{\frac{2}{3}}$ & 0.0193890739 \\
22 & 0.8539157434 & 0.9750654537 & 0.161 & $n^{\frac{2}{3}}$ & 0.0206650354 \\
23 & 0.8640994381 & 0.9758243720 & 0.161 & $n^{\frac{2}{3}}$ & 0.0173047187 \\
24 & 0.8597299761 & 0.9756198890 & 0.161 & $n^{\frac{2}{3}}$ & 0.0183709537 \\
25 & 0.8682239511 & 0.9761997433 & 0.161 & $n^{\frac{2}{3}}$ & 0.0157272307 \\
26 & 0.8646044552 & 0.9760442026 & 0.161 & $n^{\frac{2}{3}}$ & 0.0165611703 \\
27 & 0.8717111764 & 0.9764906343 & 0.161 & $n^{\frac{2}{3}}$ & 0.0144687926 \\
28 & 0.8688510450 & 0.9763842349 & 0.161 & $n^{\frac{2}{3}}$ & 0.0150699597 \\
\hline & & & & \\
\hline
\end{tabular}

\section{Appendix D.2. Exact fitting analysis of the moment of inertia eigenvalues}

In tables D3 and D4 are the fits to the smallest eigenvalue, $\lambda_{1}(n)$, of the moment of inertia matrix for the $(P-P-P)$ model using the forms (3.31) and (3.32) respectively. The form (3.31) includes $\frac{1}{n}$ type corrections while the form (3.32) includes $\frac{1}{\sqrt{n}}$ type corrections. The number of terms of the series used varied according to the number of free coefficients: e.g., if there were three free coefficients then the three terms $n, n-2$ and $n-4$ were utilized. In both cases the top of the table gives coefficients for fits where the value of $2 v_{1}$ is free, while the middle and bottom sections give coefficients that have $2 v_{1}$ fixed. We tried two fixed values: the SAW estimate of 1.19 and the best $\left\langle R_{g}^{2}\right\rangle_{n}$ differential approximant estimate for the $(P-P-P)$ model of 1.29. Coefficients from fits for $n=20$ to $n=28$ are included. The standard correction fit in table D3, with $2 v_{1}$ free, gives values of $2 v_{1}$ close to the SAW value, if slightly increasing. The coefficient, $a_{2}$, of the $\frac{1}{n}$ term is less stable if the value of $2 v_{1}$ is fixed at 1.29 than if it is fixed at 1.19 or allowed to be free. Note that the value of $a_{2}$ is only affected a little by the change from free to fixed at 1.19. All the fits in table D4 that use the $\frac{1}{\sqrt{n}}$-type corrections are less stable than those in table D3 that use the $\frac{1}{n}$ corrections. In particular, the coefficient $a_{2}$ is far less stable. This implies that the best fit concurs with the differential approximant analysis with a value of $2 v_{1}$ around 1.19 , and uses the relatively benign $\frac{1}{n}$ corrections. This second point concurs with the observation that the differential approximants of the smallest eigenvalue, $\lambda_{1}(n)$, were relatively well behaved.

In tables D5 and D6 are the fits to the largest eigenvalue, $\lambda_{3}(n)$, of the moment of inertia matrix for the $(P-P-P)$ model using the forms (3.31) and (3.32) respectively. Once again, the top of the tables give coefficients for the fits where the value of $2 \nu_{3}$ is free while the middle and bottom coefficients have $2 v_{3}$ fixed, as for $\lambda_{1}(n)$. The fit with $\frac{1}{n}$ corrections and $2 v_{3}$ free 
Table D3. The coefficients of three fits to the smallest eigenvalue, $\lambda_{1}(n)$, of the moment of inertia for the $(P-P-P)$ model. The top fit allows the value of $v$ to be free while the bottom two fits fix this exponent. These fits use $\frac{1}{n}$ corrections: see equation (3.31).

\begin{tabular}{|c|c|c|c|c|}
\hline$n$ & $a_{1}$ & $2 v_{1}$ & $a_{2}$ & $a_{3}$ \\
\hline 20 & -2.724815224 & 1.163388477 & 5.666275943 & -12.96033492 \\
\hline 21 & -2.749265119 & 1.169014693 & 5.858321147 & -13.84016515 \\
\hline 22 & -2.774577794 & 1.174725610 & 6.073152086 & -14.77803577 \\
\hline 23 & -2.793298510 & 1.178924259 & 6.233843907 & -15.61234080 \\
\hline 24 & -2.814179170 & 1.183535931 & 6.424678029 & -16.52535092 \\
\hline 25 & -2.826827330 & 1.186303333 & 6.543063127 & -17.22713264 \\
\hline 26 & -2.836927800 & 1.188491651 & 6.642284471 & -17.71643162 \\
\hline 27 & -2.846262248 & 1.190496482 & 6.735594254 & -18.32935342 \\
\hline 28 & -2.852661690 & 1.191855454 & 6.803456388 & -18.67947825 \\
\hline 20 & -2.841622425 & 1.19 & 6.621334854 & -17.22703337 \\
\hline 21 & -2.842513608 & 1.19 & 6.653550396 & -17.59298974 \\
\hline 22 & -2.843234486 & 1.19 & 6.682593176 & -17.80737537 \\
\hline 23 & -2.843624178 & 1.19 & 6.697973178 & -18.03610699 \\
\hline 24 & -2.843851650 & 1.19 & 6.708514087 & -18.07892776 \\
\hline 25 & -2.843960818 & 1.19 & 6.712785354 & -18.19870428 \\
\hline 26 & -2.843982848 & 1.19 & 6.714549189 & -18.14820023 \\
\hline 27 & -2.843920033 & 1.19 & 6.710827665 & -18.17525276 \\
\hline 28 & -2.843834255 & 1.19 & 6.707119559 & -18.05547848 \\
\hline 20 & -3.280556421 & 1.29 & 10.21020381 & -33.26010493 \\
\hline 21 & -3.286864991 & 1.29 & 10.44300945 & -35.47611016 \\
\hline 22 & -3.292724237 & 1.29 & 10.67258083 & -37.64051879 \\
\hline 23 & -3.298000706 & 1.29 & 10.88843806 & -39.91926059 \\
\hline 24 & -3.302886931 & 1.29 & 11.09941400 & -42.11210440 \\
\hline 25 & -3.307447054 & 1.29 & 11.30407740 & -44.48184698 \\
\hline 26 & -3.311730277 & 1.29 & 11.50620791 & -46.78139096 \\
\hline 27 & -3.315754269 & 1.29 & 11.70282370 & -49.25849539 \\
\hline 28 & -3.319594507 & 1.29 & 11.89941941 & -51.68867047 \\
\hline
\end{tabular}

Table D4. The coefficients of three fits to the smallest eigenvalue, $\lambda_{1}(n)$, of the moment of inertia for the $(P-P-P)$ model. The top fit allows the value of $v$ to be free while the bottom two fits fix this exponent. These fits use $\frac{1}{\sqrt{n}}$ corrections: see equation (3.32).

\begin{tabular}{lllll}
\hline$n$ & $a_{1}$ & $2 v_{1}$ & $a_{2}$ & $a_{3}$ \\
\hline 20 & -4.463970915 & 1.438199488 & -3.606651092 & 6.024597655 \\
21 & -4.417089672 & 1.430174649 & -3.480093584 & 5.893284132 \\
22 & -4.383389048 & 1.424513214 & -3.361443569 & 5.792753946 \\
23 & -4.336970494 & 1.416691312 & -3.224987032 & 5.658579334 \\
24 & -4.305279471 & 1.411481234 & -3.101773019 & 5.559966820 \\
25 & -4.251393955 & 1.402529166 & -2.928596939 & 5.399327527 \\
26 & -4.185092547 & 1.391743755 & -2.668915949 & 5.190114130 \\
27 & -4.134393805 & 1.383454870 & -2.491134144 & 5.033823177 \\
28 & -4.068857947 & 1.372925337 & -2.218436807 & 4.821717645 \\
20 & -3.002625739 & 1.19 & 0.8401583251 & 1.820134994 \\
21 & -2.989943090 & 1.19 & 1.064189417 & 1.712490790 \\
22 & -2.977800616 & 1.19 & 1.311181915 & 1.603789591 \\
23 & -2.967160395 & 1.19 & 1.519273404 & 1.508779897 \\
24 & -2.956610992 & 1.19 & 1.755659212 & 1.409638571 \\
\hline
\end{tabular}


Table D4. (continued)

\begin{tabular}{lllll}
\hline$n$ & $a_{1}$ & $2 \nu_{1}$ & $a_{2}$ & $a_{3}$ \\
\hline 25 & -2.947756459 & 1.19 & 1.945718751 & 1.326801891 \\
26 & -2.939000159 & 1.19 & 2.160325380 & 1.240761320 \\
27 & -2.931582457 & 1.19 & 2.333557705 & 1.168364089 \\
28 & -2.924319651 & 1.19 & 2.527044334 & 1.093985623 \\
20 & -3.591404322 & 1.29 & -0.9514706910 & 3.514121096 \\
21 & -3.584155248 & 1.29 & -0.8278880799 & 3.453222689 \\
22 & -3.577164741 & 1.29 & -0.6812936357 & 3.390026328 \\
23 & -3.571423036 & 1.29 & -0.5735623368 & 3.339377936 \\
24 & -3.565542637 & 1.29 & -0.4375063145 & 3.283538195 \\
25 & -3.561147627 & 1.29 & -0.3477369847 & 3.243010963 \\
26 & -3.556660462 & 1.29 & -0.2334094556 & 3.198363502 \\
27 & -3.553336603 & 1.29 & -0.1604352409 & 3.166495500 \\
28 & -3.550005621 & 1.29 & -0.0671715826 & 3.131828410 \\
\hline
\end{tabular}

Table D5. The coefficients of three fits to the larger eigenvalue, $\lambda_{3}(n)=\lambda_{2}(n)$, of the moment of inertia for the $(P-P-P)$ model. The top fit allows the value of $v$ to be free while the bottom two fits fix this exponent. These fits use $\frac{1}{n}$ corrections: see equation (3.31).

\begin{tabular}{|c|c|c|c|c|}
\hline$n$ & $a_{1}$ & $2 v_{3}$ & $a_{2}$ & $a_{3}$ \\
\hline 20 & -2.474887010 & 1.330133182 & 1.169158567 & 1.380214814 \\
\hline 21 & -2.451727355 & 1.324819993 & 0.9845802007 & 2.056262136 \\
\hline 22 & -2.424625717 & 1.318682413 & 0.7582064092 & 3.216116413 \\
\hline 23 & -2.402331687 & 1.313703614 & 0.5633338950 & 4.044177082 \\
\hline 24 & -2.384502562 & 1.309756026 & 0.4020464689 & 4.986481513 \\
\hline 25 & -2.363446512 & 1.305145685 & 0.204718825 & 5.916901080 \\
\hline 26 & -2.345549804 & 1.301270237 & 0.029444054 & 7.025885609 \\
\hline 27 & -2.327992403 & 1.297496245 & -0.146489591 & 7.927417445 \\
\hline 28 & -2.310098163 & 1.293691011 & -0.333726665 & 9.196060404 \\
\hline 20 & -1.859794757 & 1.19 & -3.860040364 & 23.84789160 \\
\hline 21 & -1.852652904 & 1.19 & -4.124366246 & 26.16626450 \\
\hline 22 & -1.846211559 & 1.19 & -4.376201889 & 28.73784289 \\
\hline 23 & -1.840251622 & 1.19 & -4.620417516 & 31.11437593 \\
\hline 24 & -1.834780204 & 1.19 & -4.856318771 & 33.76763879 \\
\hline 25 & -1.829762026 & 1.19 & -5.081959793 & 36.18085137 \\
\hline 26 & -1.825086009 & 1.19 & -5.302251764 & 38.88617400 \\
\hline 27 & -1.820788301 & 1.19 & -5.512698552 & 41.34074272 \\
\hline 28 & -1.816777611 & 1.19 & -5.717671690 & 44.07061471 \\
\hline 20 & -2.298728754 & 1.29 & -0.2711713545 & 7.814819509 \\
\hline 21 & -2.297004286 & 1.29 & -0.3349072110 & 8.283144238 \\
\hline 22 & -2.295701309 & 1.29 & -0.3862142636 & 8.904699700 \\
\hline 23 & -2.294628149 & 1.29 & -0.4299526474 & 9.231222466 \\
\hline 24 & -2.293815488 & 1.29 & -0.4654187863 & 9.734461374 \\
\hline 25 & -2.293248258 & 1.29 & -0.4906678487 & 9.897709787 \\
\hline 26 & -2.292833438 & 1.29 & -0.5105930268 & 10.25298309 \\
\hline 27 & -2.292622533 & 1.29 & -0.5207026654 & 10.25750186 \\
\hline 28 & -2.292537860 & 1.29 & -0.5253719254 & 10.43742375 \\
\hline
\end{tabular}

(table D5) is clearly unstable with $2 v_{3}$ decreasing and the coefficient $a_{2}$ changing rapidly. Furthermore, by fixing the value of $2 v_{3}$ to 1.29 , which is the same as the free estimate of around 1.29 , a big change is produced in the coefficient $a_{2}$. Better behaved are some of the fits 
Table D6. The coefficients of three fits to the largest eigenvalue, $\lambda_{3}(n)=\lambda_{2}(n)$, of the moment of inertia for the $(P-P-P)$ model. The top fit allows the value of $v$ to be free while the bottom two fits fix this exponent. These fits use $\frac{1}{\sqrt{n}}$ corrections: see equation (3.32).

\begin{tabular}{|c|c|c|c|c|}
\hline$n$ & $a_{1}$ & $2 v_{3}$ & $a_{2}$ & $a_{3}$ \\
\hline 20 & -2.289709609 & 1.300873021 & 2.156581910 & -0.641494007 \\
\hline 21 & -2.203913210 & 1.286015179 & 2.372074485 & -0.875640625 \\
\hline 22 & -2.074442935 & 1.264311462 & 2.811630202 & -1.260842112 \\
\hline 23 & -2.002427148 & 1.252107081 & 3.013642266 & -1.465889862 \\
\hline 24 & -1.934589619 & 1.240977908 & 3.276552782 & -1.677637868 \\
\hline 25 & -1.874144773 & 1.230877504 & 3.457941812 & -1.854517812 \\
\hline 26 & -1.810893738 & 1.220664223 & 3.722052864 & -2.058290520 \\
\hline 27 & -1.770887298 & 1.214043641 & 3.844019958 & -2.177090231 \\
\hline 28 & -1.711412712 & 1.204558139 & 4.107592804 & -2.373584861 \\
\hline 20 & -1.636913110 & 1.19 & 4.143013210 & -2.519666678 \\
\hline 21 & -1.633379274 & 1.19 & 4.188757262 & -2.547008047 \\
\hline 22 & -1.629046469 & 1.19 & 4.292272220 & -2.588222694 \\
\hline 23 & -1.627137319 & 1.19 & 4.313440217 & -2.602820328 \\
\hline 24 & -1.624169435 & 1.19 & 4.394573742 & -2.632908767 \\
\hline 25 & -1.623405873 & 1.19 & 4.395447180 & -2.637815348 \\
\hline 26 & -1.621492277 & 1.19 & 4.456090152 & -2.658581094 \\
\hline 27 & -1.621394660 & 1.19 & 4.443674362 & -2.657516847 \\
\hline 28 & -1.620325282 & 1.19 & 4.485241679 & -2.670248710 \\
\hline 20 & -2.225691696 & 1.29 & 2.351384151 & -0.8256805566 \\
\hline 21 & -2.227591431 & 1.29 & 2.296679775 & -0.8062761517 \\
\hline 22 & -2.228410599 & 1.29 & 2.299796592 & -0.8019859233 \\
\hline 23 & -2.231399964 & 1.29 & 2.220604371 & -0.7722222431 \\
\hline 24 & -2.233101073 & 1.29 & 2.201408356 & -0.7590092032 \\
\hline 25 & -2.236797036 & 1.29 & 2.101991533 & -0.7216063134 \\
\hline 26 & -2.239152583 & 1.29 & 2.062355258 & -0.7009788882 \\
\hline 27 & -2.243148807 & 1.29 & 1.949681393 & -0.6593854256 \\
\hline 28 & -2.246011252 & 1.29 & 1.891025753 & -0.6324059189 \\
\hline
\end{tabular}

in table D6, with the fit using $2 v_{3}$ free and that fixing it at 1.19 giving similar results, although not as good as in the $\lambda_{1}(n)$ analysis. So we can tentatively conclude that, opposed to the differential approximant analysis for $\lambda_{3}(n)$, an estimate of $2 \nu_{3}$ around the SAW value of 1.19, with strong $\frac{1}{\sqrt{n}}$ corrections, is best supported by the data fits. While somewhat debatable, it is certainly consistent with the data fits.

\section{References}

[1] Madras N and Slade G 1993 The Self-Avoiding Walk (Boston: Birkhauser)

[2] Redner S and Majid I 1983 J. Phys. A: Math. Gen. 16 L307

[3] Privman V and Švrakić N M 1989 Directed Models of Polymers, Interfaces, and Clusters: Scaling and Finite-Size Properties (Lecture Notes in Physics vol 338) (Berlin: Springer)

[4] Grassberger P 1982 Z. Phys. B 48255

[5] Guttmann A J and Wormald N 1984 J. Phys. A: Math. Gen. 17 L271

[6] Joyce G S 1984 J. Phys. A: Math. Gen. 17 L463

[7] Whittington S G 1984 J. Phys. A: Math. Gen. 17 L117

[8] Blöte H W J and Hilhorst H J 1984 J. Phys. A: Math. Gen. 17 L111

[9] Manna S S 1984 J. Phys. A: Math. Gen. 17 L899

[10] Whittington S G 1985 J. Phys. A: Math. Gen. 18 L67

[11] Guttmann A J and Wallace K J 1986 J. Phys. A: Math. Gen. 191645 
[12] Guttmann A J, Prellberg T and Owczarek A L 1993 J. Phys. A: Math. Gen. 266615

[13] Brak R, Owczarek A L and Soteros C 1998 J. Phys. A: Math. Gen. 314851

[14] Guttmann A J and Wallace K J 1985 J. Phys. A: Math. Gen. 18 L1049

[15] Enting I G and Guttmann A J 1989 J. Phys. A: Math. Gen. 221371

[16] Guttmann A J 1989 J. Phys. A: Math. Gen. 222807

[17] Guttmann A J and Wang J 1991 J. Phys. A: Math. Gen. 243107

[18] Joyce G S and Brak R 1985 J. Phys. A: Math. Gen. 18 L293

[19] Liu K C and Lin K Y 1985 J. Phys. A: Math. Gen. 18 L647

[20] Szekeres G and Guttmann A J 1987 J. Phys. A: Math. Gen. 20481

[21] Nienhuis B 1982 Phys. Rev. Lett. 491062

[22] Fisher M E and Sykes M F 1959 Phys. Rev. 11445

[23] Li B, Madras N and Sokal A D 1995 J. Stat. Phys. 80661

[24] Caracciolo S, Causo M S and Pelissetto A 1998 Phys. Rev. E 57 R1215

[25] Guttmann A J 1989 Phase Transitions and Critical Phenomena vol 13, ed C Domb and J L Lebowitz (New York: Academic)

[26] Batchelor M T, Bennett-Wood D and Owczarek A L 1998 Eur. Phys. J. B 5139

[27] Andrews G E 1976 The theory of partitions Encyclopedia of Mathematics and its Applications vol 2, ed G-C Rata (Reading, MA: Addison-Wesley)

[28] Bhatia D P, Prasad M A and Arora D 1997 J. Phys. A: Math. Gen. 302281

[29] Conway A and Guttmann A J 1996 Phys. Rev. Lett. 775284

[30] Anonymous referee 2000 private communication

[31] Owczarek A L, Rechnitzer A and Wong L H 2000 The anti-spiral self-avoiding walk, in preparation

[32] Rechnitzer A 2000 PhD Thesis University of Melbourne, in preparation

[33] MacMahon P A 1960 Combinatory Analysis vol 2 (New York: Chelsea) section 8 